\title{
Tri-level decision-making with multiple followers: Model, algorithm and case study
}

\author{
Jialin Han ${ }^{\mathrm{a}, \mathrm{b}}$, Jie Lu ${ }^{\mathrm{b},}$, , Yaoguang Hu $\mathrm{u}^{\mathrm{a}}$, Guangquan Zhang ${ }^{\mathrm{b}}$, \\ ${ }^{a}$ Industrial and Systems Engineering Laboratory, School of Mechanical Engineering, Beijing Institute of \\ Technology, China \\ ${ }^{\mathrm{b}}$ Decision Systems and e-Service Intelligence Laboratory, Centre for Quantum Computation \& Intelligent \\ Systems, Faculty of Engineering and Information Technology, University of Technology Sydney, Australia \\ E-mail addresses: hj1@bit.edu.cn (J. Han),jie.lu@uts.edu.au (J. Lu), \\ hyg@bit.edu.cn (Y. Hu), guangquan.zhang@uts.edu.au (G. Zhang). \\ * Corresponding author at: Faculty of Engineering and Information Technology, University of Technology \\ Sydney, PO Box 123, Broadway, NSW 2007, Australia. Tel.: +61-2-95141838.
}

\begin{abstract}
Tri-level decision-making arises to address compromises among interacting decision entities distributed throughout a three-level hierarchy; these entities are respectively termed the top-level leader, the middle-level follower and the bottom-level follower. This study considers an uncooperative situation where multiple followers at the same (middle or bottom) level make their individual decisions independently but consider the decision results of their counterparts as references through information exchanged among themselves. This situation is called a reference-based uncooperative multi-follower tri-level (MFTL) decision problem which appears in many real-world applications. To solve this problem, we need to find an optimal solution achieving both the Stackelberg equilibrium in the three-level vertical structure and the Nash equilibrium among multiple followers at the same horizontal level. In this paper, we first propose a general linear MFTL decision model for this situation. We then develop a MFTL Kth-Best algorithm to find an optimal solution to the model. Since the optimal solution means a compromised result in the uncooperative situation and it is often imprecise or ambiguous for decision entities to identify their related satisfaction, we use a fuzzy programming approach to characterize and evaluate the solution obtained. Lastly, a real-world case study on production-inventory planning illustrates the effectiveness of the proposed MFTL decision techniques.
\end{abstract}

Keywords: Tri-level decision-making; multilevel programming; Kth-Best algorithm; fuzzy programming; production-inventory planning.

\section{Introduction}

Tri-level decision-making (also known as tri-level programming) technique has been developed to deal with decentralized decision problems involving interacting decision entities that are distributed throughout a three-level hierarchy, which is a subfamily of multilevel programming [30] motivated by Stackelberg game theory [26]. Decision entities at the three hierarchical levels are respectively termed the top-level leader, the middle-level follower and the bottom-level follower. The decision entities make their individual decisions in sequence, from the top level to the middle level and then to the bottom level with the aim of optimizing their respective objectives [36]. Specifically, the leader gives priority to making a decision; however, this decision 
is implicitly determined by the actions of the followers. The middle-level follower then reacts to the decision made by the leader and optimizes its own objective function while taking into account the implicit reactions of the bottom-level follower. Lastly, in view of the given decisions from the top and middle levels, the bottom-level follower makes decision to optimize its own objective function. The decision process is repeatedly executed until the Stackelberg equilibrium is achieved in the three-level vertical structure, which differs from the traditional Stackelberg game where the decisions made by the followers do not affect the decision, which has been already taken by the leader [11]. This category of the hierarchical decision-making process often appears in many decentralized management problems in applications, such as supply chain management [33], resource allocation optimization [20, 34] and hierarchical production operations [29].

The hierarchical production-inventory planning in a conglomerate enterprise can be taken as an example. The conglomerate is composed of a sales company, a logistics center and a manufacturing factory, which are distributed throughout a three-stage supply chain. To fully satisfy market demand and shorten time-to-market, the sales company and the logistics center have to hold a certain amount of inventory using their respective warehouses but both of them nonetheless seek to minimize their individual inventory holding costs. When making the production-inventory plan within a stable sales cycle, the sales company (the leader) takes the lead in developing an optimal inventory plan which considers the current market demand and implicit reactions of other decision entities. The logistics center (the middle-level follower) then makes an optimal inventory plan under the decision given by the sales company and considers the implicit production planning of the manufacturing factory (the bottom-level follower). Lastly, the manufacturing factory makes the production plan to minimize its own cost of production in light of the fixed inventory plans. The decision process will not stop until the Stackelberg equilibrium among the decision entities is achieved. Consequently, the example describes a typical tri-level decision-making problem in which decisions are sequentially and repeatedly executed with all decision entities seeking to optimize their individual objectives until the Stackelberg equilibrium is achieved.

In general, there are two fundamental issues in supporting such a tri-level decision-making process. One is how to use a model to describe the decision-making process, which may manifest different characteristics at the three decision levels, and the other is how to find an optimal solution to the problem. Whereas the majority of studies on multilevel programming were focused on bi-level decision-making (also known as bi-level programming) such as in $[3,5,9,10,12,14,17,31,35]$, research on tri-level decision-making has increasingly attracted investigations into decision models, solution algorithms and applications since it can be used to deal with many decentralized decision problems in the real world. Bard [4] first presented an investigation of linear tri-level programming and designed a cutting plane algorithm to solve such problems, based on which White [32] proposed a penalty function approach for linear tri-level programming problems. Faísca, Saraiva, Rustem and Pistikopoulos [11] studied a multi-parametric programming approach to solve tri-level hierarchical and decentralized optimization problems. Yao, Edmunds, Papageorgiou and Alvarez [34] built a tri-level optimization model for resource allocation in electric power network defense and proposed a decomposition approach to find an optimal solution to the model. Recently, Alguacil, Delgadillo and Arroyo [1] adopted a tri-level decision model to describe an electric grid defense planning problem and 
solved it using a novel two-stage solution approach. Street, Moreira and Arroyo [27] developed a tri-level decision model for energy reserve scheduling in electricity markets with transmission flow limits and found a solution to it by a Benders decomposition approach.

Although numerous studies have been carried out, existing tri-level decision-making research has been primarily limited to a specific situation in which one single decision entity is involved at each level. However, more decision entities are often involved at the middle and bottom levels in a tri-level decision-making case; these entities are called multiple followers. In the production-inventory planning example, the sales company (the leader) may have several subordinate logistics centers (the middle-level followers) and there may also be several manufacturing factories (the bottom-level followers) attached to each logistics center. Moreover, multiple followers at the same level may have a variety of relationships with one another. In our previous research [18], we developed 64 kinds of standard situations to describe various relationships within multi-follower tri-level (MFTL) decision problems, such as the uncooperative relationship, cooperative relationship, and semi-cooperative relationship. Such diverse relationships among multiple followers will generate different decision processes which need to be described and solved using different decision models and solution methods. As almost no research on MFTL decision-making has been proposed apart from some limited discussion about programming models [18, 23], further investigation into MFTL decision models together with solution methods is necessary and urgent. Furthermore, MFTL decision techniques in real-world applications are crucially required.

This study considers an uncooperative situation where multiple followers at the same level make their individual decisions independently but exchange information among themselves, which implies that followers consider the decision results of their counterparts as references when making their individual decisions. The situation is known as a reference-based uncooperative (or reference-uncooperative) relationship, which is very common and popular among competitive or uncooperative decision entities in some hierarchical organizations. For example, in the proposed production-inventory planning instance, the independent logistics centers and factories may reference inventory or production plans determined by their counterparts at the same level when making their individual decisions. More specifically, within MFTL decision-making, if multiple followers at the same level determine their individual decision variables independently but simultaneously take the decision results of their counterparts for references to optimize their respective objectives, this can be called a reference-uncooperative MFTL decision problem. Solving this kind of MFTL decision problem implies that we need to find an optimal solution known as a Stackelberg-Nash solution to achieve not only the Stackelberg equilibrium in the tri-level vertical structure but also the Nash equilibrium among multiple followers at the same horizontal level. To support such a decision-making process, this paper will model this reference-uncooperative MFTL decision situation and find an optimal solution to the model.

In addition, the optimal solution only means a compromised result for a MFTL decision problem, which cannot reflect the operations of the complex MFTL decision-making process completely; that is, it is imprecise or ambiguous for decision entities to evaluate the solution obtained whether or not they desire to in real-world cases. It is necessary to find a practical way to identify the satisfaction of decision entities towards 
the solution obtained by the MFTL Kth-Best algorithm. In terms of related research, Lai [15], Shih, Lai and Lee [23] and Sinha [24, 25] have developed fuzzy approaches to identify the satisfaction of decision entities and have obtained solutions to multilevel programming problems. Pramanik and Roy [21] have proposed another fuzzy approach using linear goal programming to solve such problems. However, these fuzzy approaches are limited to a special situation where decision entities from different levels share the same constraint conditions with each other and the solutions obtained are not the compromised equilibrium. This study will overcome this existing issue and extend these fuzzy programming approaches to evaluate the solution obtained and analyze the decision-making process in the uncooperative MFTL decision situation.

The main contribution of this paper is twofold. First, it provides a general decision model and a solution method with related algorithm to solve reference-uncooperative MFTL decision problems; and second, it adopts a fuzzy programming approach to identify the satisfaction of decision entities towards the solution obtained. We first present a linear MFTL decision model for the reference-uncooperative situation and discusses related theoretical properties of the model. A MFTL Kth-Best algorithm is then developed to find an optimal solution to the MFTL decision model and a fuzzy programming approach is used to evaluate the solution obtained. Lastly, a detailed case study on production-inventory planning illustrates the proposed MFTL decision-making techniques in applications.

The paper is organized as follows. Following the introduction, a general linear reference-uncooperative MFTL decision model along with related theoretical properties are presented in Section 2. In Section 3, a MFTL Kth-Best algorithm is proposed to find an optimal solution to the model. We then use a fuzzy programming approach to analyze the satisfactory degree of decision entities towards obtained solutions in Section 4. A case study and a related decision support system on production-inventory planning illustrate our research in Section 5. Lastly, in Section 6, concluding remarks and further avenues of study are given.

\section{Multi-follower tri-level decision model and related properties}

This section will present the developed reference-uncooperative MFTL decision model and discuss related theoretical properties.

\subsection{A general reference-uncooperative MFTL decision model and related solution concepts}

The organizational structure among decision entities in the three-level hierarchy that is studied in this paper is shown as Fig. 1.

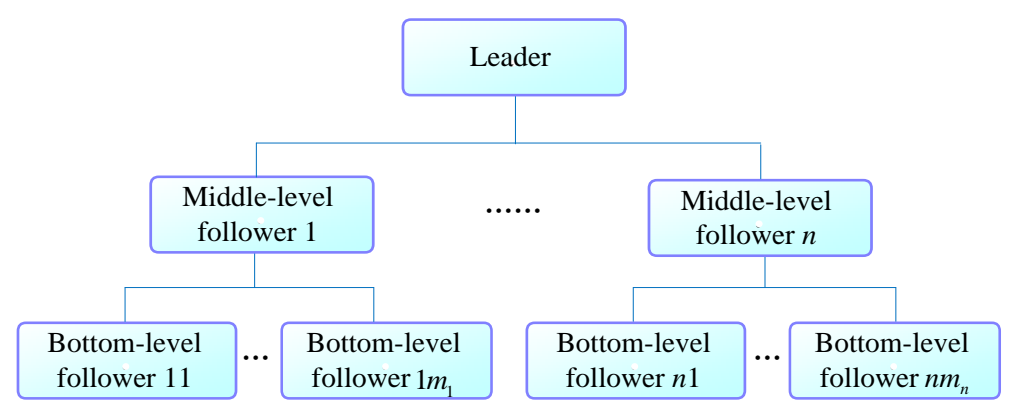

Fig. 1. The organizational structure of the three-level hierarchy 
Let $x \in X \subset R^{k}, y_{i} \in Y_{i} \subset R^{k_{i}}, z_{i j} \in Z_{i j} \subset R^{k_{i j}}$ denote the vectors of decision variables of the leader, the middle-level follower $i$, and the bottom-level follower $i j$ respectively where $j=1,2, \ldots, m_{i}, i=1,2, \ldots, n$. We give detailed definitions of the reference-uncooperative relationship as follows.

Definition 2.1 If the decision variables $y_{1}, \ldots, y_{i-1}, y_{i+1}, \ldots, y_{n}$ controlled by the counterparts of the middle-level follower $i$ are involved in its the objective function and constraint conditions apart from its own decision variable $y_{i}$ and the decision variables $x, z_{i 1}, \ldots, z_{i m_{i}}$ determined by the leader and the bottom-level followers, this is a reference-uncooperative relationship among multiple followers at the middle level.

Definition 2.2 If the decision variables $z_{i 1}, \ldots, z_{i(j-1)}, z_{i(j+1)}, \ldots, z_{i m_{i}}$ controlled by the counterparts of the bottom-level follower $i j$ are involved in its objective function and constraint conditions apart from its own decision variable $z_{i j}$ and the decision variables $x$ and $y_{i}$ respectively determined by the leader and the middle-level follower $i$, this can be called a reference-uncooperative relationship among multiple bottom-level followers attached to the same middle-level follower $i$.

As we can see from Definitions 2.1 and 2.2, the reference-uncooperative relationship implies that each decision entity at the middle or bottom level should consider decision results made by its counterparts as references when determining its own decision variable to optimize its individual objective function. Based on the definitions, we propose a general linear MFTL decision model together with the reference-uncooperative relationship among both middle-level and bottom-level followers.

For $\quad x \in X \subset R^{k} \quad, \quad y_{i} \in Y_{i} \subset R^{k_{i}} \quad, \quad z_{i j} \in Z_{i j} \subset R^{k_{i j}} \quad, \quad f^{(1)}: X \times Y_{1} \times \ldots \times Y_{n} \times Z_{11} \times \ldots \times Z_{1 m_{1}} \times \ldots \times Z_{n m_{n}} \rightarrow R^{1} \quad$, $f_{i}^{(2)}: X \times Y_{1} \times \ldots \times Y_{n} \times Z_{i 1} \times \ldots \times Z_{i m_{i}} \rightarrow R^{1}, f_{i j}^{(3)}: X \times Y_{i} \times Z_{i 1} \times \ldots \times Z_{i m_{i}} \rightarrow R^{1}, \quad j=1,2, \ldots, m_{i}, i=1,2, \ldots, n, \quad$ a linear reference-uncooperative MFTL decision model in which one leader, $n$ middle-level followers and $m_{i}$ bottom-level followers attached to the middle-level follower $i$ are involved is defined as follows:

$$
\begin{aligned}
& \min _{x \in X} f^{(1)}\left(x, y_{1}, \ldots, y_{n}, z_{11}, \ldots, z_{1 m_{1}}, \ldots, z_{n 1}, \ldots, z_{n m_{n}}\right)=c x+\sum_{i=1}^{n} d_{i} y_{i}+\sum_{i=1}^{n} \sum_{j=1}^{m_{i}} e_{i j} z_{i j} \\
& \text { s.t. } \quad A x+\sum_{i=1}^{n} B_{i} y_{i}+\sum_{i=1}^{n} \sum_{j=1}^{m_{i}} C_{i j} z_{i j} \leq b,
\end{aligned}
$$

where $y_{i}, z_{i 1}, \ldots, z_{i m_{i}}(i=1,2, \ldots, n)$, for the given $\left(x, y_{1}, \ldots, y_{i-1}, y_{i+1}, \ldots, y_{n}\right)$, solve (1c-1f):

$$
\begin{aligned}
& \min _{y_{i} \in Y_{i}} f_{i}^{(2)}\left(x, y_{1}, \ldots, y_{n}, z_{i 1}, \ldots, z_{i m_{i}}\right)=c_{i} x+\sum_{s=1}^{n} g_{i s} y_{s}+\sum_{j=1}^{m_{i}} h_{i j} z_{i j} \\
& \text { s.t. } \quad A_{i} x+\sum_{s=1}^{n} D_{i s} y_{s}+\sum_{j=1}^{m_{i}} E_{i j} z_{i j} \leq b_{i},
\end{aligned}
$$

where $z_{i j}\left(j=1,2, \ldots, m_{i}\right)$, for the given $\left(x, y_{i}, z_{i 1}, \ldots, z_{i(j-1)}, z_{i(j+1)}, \ldots, z_{i m_{i}}\right)$, solves $(1 \mathrm{e}-1 \mathrm{f})$ :

$$
\begin{aligned}
& \min _{z_{i j} \in Z_{i j}} f_{i j}^{(3)}\left(x, y_{i}, z_{i 1}, \ldots, z_{i m_{i}}\right)=c_{i j} x+p_{i j} y_{i}+\sum_{t=1}^{m_{i}} q_{i j t} z_{i t} \\
& \text { s.t. } \quad A_{i j} x+P_{i j} y_{i}+\sum_{t=1}^{m_{i}} Q_{i j t} z_{i t} \leq b_{i j}
\end{aligned}
$$


where $c, c_{i}, c_{i j} \in R^{k}, d_{i}, p_{i j} \in R^{k_{i}}, g_{i s} \in R^{k_{s}}, e_{i j}, h_{i j} \in R^{k_{i j}}, q_{i j t} \in R^{k_{i t}}, A \in R^{r \times k}, A_{i} \in R^{r_{i} \times k}, A_{i j} \in R^{r_{i j} \times k}, B_{i} \in R^{r \times k_{i}}$, $D_{i s} \in R^{r_{i} \times k_{s}}, P_{i j} \in R^{r_{i j} \times k_{i}}, C_{i j} \in R^{r \times k_{i j}}, E_{i j} \in R^{r_{i} \times k_{i j}}, Q_{i j t} \in R^{r_{i j} \times k_{i j}}, b \in R^{r}, b_{i} \in R^{r_{i}}, b_{i j} \in R^{r_{i j}}$ for $\quad j=1,2, \ldots, m_{i}, t=1,2, \ldots, m_{i}$, $i=1,2, \ldots, n, s=1,2, \ldots, n$.

To find a Stackbelberg-Nash solution to the MFTL decision model (1), relevant solution concepts are defined as follows based on the MFTL hierarchical structure.

\section{Definition 2.3}

(a) Constraint region of the MFTL decision model (1):

$$
\begin{aligned}
& S=\left\{\left(x, y_{1}, \ldots, y_{n}, z_{11}, \ldots, z_{1 m_{1}}, \ldots, z_{n 1}, \ldots, z_{n m_{n}}\right)\right. \in X \times \prod_{i=1}^{n} Y_{i} \times \prod_{i=1}^{n} \prod_{j=1}^{m_{i}} Z_{i j}: A x+\sum_{i=1}^{n} B_{i} y_{i}+\sum_{i=1}^{n} \sum_{j=1}^{m_{i}} C_{i j} z_{i j} \leq b, \\
&\left.A_{i} x+\sum_{s=1}^{n} D_{i s} y_{s}+\sum_{j=1}^{m_{i}} E_{i j} z_{i j} \leq b_{i}, A_{i j} x+P_{i j} y_{i}+\sum_{i=1}^{m_{i}} Q_{i j t} z_{i t} \leq b_{i j}, j=1,2, \ldots, m_{i}, i=1,2, \ldots, n\right\} .
\end{aligned}
$$

(b) Feasible set of the middle-level follower $i(i=1,2, \ldots, n)$ and its bottom-level followers:

$$
S_{i}\left(x, y_{1}, \ldots, y_{i-1}, y_{i+1}, \ldots, y_{n}\right)=\left\{\left(y_{i}, z_{i 1}, \ldots, z_{i m_{i}}\right) \in Y_{i} \times \prod_{j=1}^{m_{i}} Z_{i j}: A_{i} x+\sum_{s=1}^{n} D_{i s} y_{s}+\sum_{j=1}^{m_{i}} E_{i j} z_{i j} \leq b_{i}, A_{i j} x+P_{i j} y_{i}+\sum_{i=1}^{m_{i}} Q_{i j t} z_{i t} \leq b_{i j}, j=1,2, \ldots, m_{i}\right\} .
$$

(c) Feasible set of the bottom-level follower $i j \quad\left(j=1,2, \ldots, m_{i}, i=1,2, \ldots, n\right)$ :

$$
S_{i j}\left(x, y_{i}, z_{i 1}, \ldots, z_{i(j-1)}, z_{i(j+1)}, \ldots, z_{i m_{i}}\right)=\left\{z_{i j} \in Z_{i j}: A_{i j} x+P_{i j} y_{i}+\sum_{t=1}^{m_{i}} Q_{i j t} z_{i t} \leq b_{i j}\right\} .
$$

(d) Rational reaction set of the bottom-level follower $i j\left(j=1,2, \ldots, m_{i}, i=1,2, \ldots, n\right)$ :

$$
\begin{gathered}
P_{i j}\left(x, y_{i}, z_{i 1}, \ldots, z_{i(j-1)}, z_{i(j+1)}, \ldots, z_{i m_{i}}\right)=\left\{z_{i j} \in Z_{i j}: z_{i j} \in \arg \min \left[f_{i j}^{(3)}\left(x, y_{i}, z_{i 1}, \ldots, z_{i(j-1)}, \hat{z}_{i j}, z_{i(j+1)}, \ldots, z_{i m_{i}}\right),\right.\right. \\
\left.\left.\hat{z}_{i j} \in S_{i j}\left(x, y_{i}, z_{i 1}, \ldots, z_{i(j-1)}, z_{i(j+1)}, \ldots, z_{i m_{i}}\right)\right]\right\} .
\end{gathered}
$$

(e) Rational reaction set of all the bottom-level followers attached to the middle-level follower $i$ $(i=1,2, \ldots, n)$ :

$P_{i}\left(x, y_{i}\right)=\left\{\left(z_{i 1}, \ldots, z_{i m_{i}}\right) \in \prod_{j=1}^{m_{i}} Z_{i j}: z_{i j} \in P_{i j}\left(x, y_{i}, z_{i 1}, \ldots, z_{i(j-1)}, z_{i(j+1)}, \ldots, z_{i m_{i}}\right), j=1,2, \ldots, m_{i}\right\}$.

(f) Rational reaction set of the middle-level follower $i(i=1,2, \ldots, n)$ and its bottom-level followers:

$$
\begin{array}{r}
P_{i}\left(x, y_{1}, \ldots, y_{i-1}, y_{i+1}, \ldots, y_{n}\right)=\left\{\left(y_{i}, z_{i 1}, \ldots, z_{i m_{i}}\right) \in Y_{i} \times \prod_{j=1}^{m_{i}} Z_{i j}:\left(y_{i}, z_{i 1}, \ldots, z_{i m_{i}}\right) \in \arg \min \left[f _ { i } ^ { ( 2 ) } \left(x, y_{1}, \ldots, y_{i-1}, \hat{y}_{i}, y_{i+1}, \ldots,\right.\right.\right. \\
\left.\left.\left.y_{n}, \hat{z}_{i 1}, \ldots, \hat{z}_{i m_{i}}\right):\left(\hat{y}_{i}, \hat{z}_{i 1}, \ldots, \hat{z}_{i m_{i}}\right) \in S_{i}\left(x, y_{1}, \ldots, y_{i-1}, y_{i+1}, \ldots, y_{n}\right),\left(\hat{z}_{i 1}, \ldots, \hat{z}_{i m_{i}}\right) \in P_{i}\left(x, \hat{y}_{i}\right)\right]\right\} .
\end{array}
$$

(g) Inducible region (IR) of model (1):

$$
\begin{array}{r}
I R=\left\{\left(x, y_{1}, \ldots, y_{n}, z_{11}, \ldots, z_{1 m_{1}}, \ldots, z_{n m_{1}}, \ldots, z_{n m_{n}}\right):\left(x, y_{1}, \ldots, y_{n}, z_{11}, \ldots, z_{1 m_{1}}, \ldots, z_{n m_{1}}, \ldots, z_{n m_{n}}\right) \in S,\right. \\
\left.\left(y_{i}, z_{i 1}, \ldots, z_{i m_{i}}\right) \in P_{i}\left(x, y_{1}, \ldots, y_{i-1}, y_{i+1}, \ldots, y_{n}\right), i=1,2, \ldots, n\right\} .
\end{array}
$$

(h) Optimal solution set of model (1):

$O S=\left\{\left(x, y_{1}, \ldots, y_{n}, z_{11}, \ldots, z_{1 m_{1}}, \ldots, z_{n m_{1}}, \ldots, z_{n m_{n}}\right):\left(x, y_{1}, \ldots, y_{n}, z_{11}, \ldots, z_{1 m_{1}}, \ldots, z_{n m_{1}}, \ldots, z_{n m_{n}}\right) \in \arg \min [\right.$

$$
\left.\left.f^{(1)}\left(x, y_{1}, \ldots, y_{n}, z_{11}, \ldots, z_{1 m_{1}}, \ldots, z_{n 1}, \ldots, z_{n m_{n}}\right):\left(x, y_{1}, \ldots, y_{n}, z_{11}, \ldots, z_{1 m_{1}}, \ldots, z_{n 1}, \ldots, z_{n m_{n}}\right) \in I R\right]\right\} .
$$


Based on related solution concepts, it can be concluded that the reference-uncooperative MFTL decision model has the following features: (1) there are reference-uncooperative relationships among both multiple middle-level followers and multiple bottom-level followers attached to the same middle-level follower; (2) the leader has the priority to determine its decision variable $x$ to optimize its objective function under the constraint region $S$; (3) the middle-level follower $i$ then determines its individual decision variable $y_{i}$ under the feasible set $S_{i}\left(x, y_{1}, \ldots, y_{i-1}, y_{i+1}, \ldots, y_{n}\right)$ to react to the given decision $\left(x, y_{1}, \ldots, y_{i-1}, y_{i+1}, \ldots, y_{n}\right)$ from the leader and other middle-level followers; (4) the bottom-level follower $i j$ determines its decision variable $z_{i j}$ under its feasible set $S_{i j}\left(x, y_{i}, z_{i 1}, \ldots, z_{i(j-1)}, z_{i(j+1)}, \ldots, z_{i m_{i}}\right)$ to respond to the decision $\left(x, y_{i}, z_{i 1}, \ldots, z_{i(j-1)}, z_{i(j+1)}, \ldots, z_{i m_{i}}\right)$ made by the leader, the middle-level follower $i$ and its own counterparts; (5) since each decision entity seeks to optimize its own objective function, the decision variable selection of the bottom-level follower $i j$ must be involved in its rational reaction set $P_{i j}\left(x, y_{i}, z_{i 1}, \ldots, z_{i(j-1)}, z_{i(j+1)}, \ldots, z_{i m_{i}}\right)$, which ensures an optimal solution to problem (1e-1f) under the given decision $\left(x, y_{i}, z_{i 1}, \ldots, z_{i(j-1)}, z_{i(j+1)}, \ldots, z_{i m_{i}}\right) ;(6)$ as the decision of the middle-level follower $i$ is affected by actions of its bottom-level followers, it must consider implicit reactions of its bottom-level followers when making its own decisions, thus, a Stackelberg-Nash solution $\left(y_{i}, z_{i 1}, \ldots, z_{i m_{i}}\right)$ to the middle-level follower $i$ and its bottom-level followers must occur in their rational reaction set $P_{i}\left(x, y_{1}, \ldots, y_{i-1}, y_{i+1}, \ldots, y_{n}\right)$, which can also be considered as an optimal solution to problem (1c-1f) under the given decision $\left(x, y_{1}, \ldots, y_{i-1}, y_{i+1}, \ldots, y_{n}\right) ;(7)$ a Stackelberg-Nash solution to all the followers under the given $x$ by the leader must be involved in $P(x)=\left\{\left(y_{1}, \ldots, y_{n}, z_{11}, \ldots, z_{1 m_{1}}, \ldots, z_{n 1}, \ldots, z_{n m_{n}}\right):\left(y_{i}, z_{i 1}, \ldots, z_{i m_{i}}\right) \in P_{i}\left(x, y_{1}, \ldots, y_{i-1}, y_{i+1}, \ldots, y_{n}\right), i=1,2, \ldots, n\right\} ;$ (8) as the leader should consider implicit reactions of all the followers when making its own decisions, an optimal solution (also known as a Stackelberg-Nash solution) to model (1) must occur over the inducible region (IR) and the optimal solution set is expressed by $O S$.

\subsection{Related theoretical properties}

For the sake of developing an efficient algorithm to solve the MFTL decision model (1), we now turn our attention to the geometry of the solution space shown as the following theoretical properties of model (1). To ensure that the model (1) is well posed, it is necessary to make some assumptions about the MFTL decision model as the basis for the existence of solutions.

(1) $S$ is nonempty and compact.

(2) IR is nonempty.

(3) $P_{i}\left(x, y_{i}\right)$ and $P(x)$ have at most one solution respectively for each parameter $\left(x, y_{i}\right)$ and $x$, where $i=1,2, \ldots, n$.

Theorem 2.1 If the above assumptions (1-3) hold, there exists an optimal solution to the MFTL decision model (1).

Proof. Since both $S$ and $I R$ are not empty, there is at least one parameter value $x^{*}$ and $P\left(x^{*}\right) \neq \varnothing$. Consider 
a sequence $\left\{\left(x^{t}, y_{1}^{t}, \ldots, y_{n}^{t}, z_{11}^{t}, \ldots, z_{1 m_{1}}^{t}, \ldots, z_{n 1}^{t}, \ldots, z_{n m_{n}}^{t}\right)\right\}_{t=1}^{\infty} \subseteq I R$ converging to $\left(x^{*}, y_{1}^{*}, \ldots, y_{n}^{*}, z_{11}^{*}, \ldots, z_{1 m_{1}}^{*}, \ldots\right.$, $\left.z_{n 1}^{*}, \ldots, z_{n m_{n}}^{*}\right)$. Then, by the well-known results of linear parametric optimization, we have $\left(y_{1}^{*}, \ldots, y_{n}^{*}, z_{11}^{*}, \ldots\right.$, $\left.z_{1 m_{1}}^{*}, \ldots, z_{n 1}^{*}, \ldots, z_{n m_{n}}^{*}\right) \in P\left(x^{*}\right)$. Hence, $I R$ is closed. Also, $I R$ is bounded by $I R \subseteq S$ and assumption (1). Therefore, the inducible region $I R$ is a nonempty and compact set. Furthermore, by assumption (3), the Nash equilibrium $\left(z_{i 1}^{*}, \ldots, z_{i m_{i}}^{*}\right)$ among the bottom-level followers attached to the same middle-level follower $i$ $(i=1,2, \ldots, n)$ and the Stackelberg-Nash equilibrium $\left(y_{1}^{*}, \ldots, y_{n}^{*}, z_{11}^{*}, \ldots, z_{1 m_{1}}^{*}, \ldots, z_{n 1}^{*}, \ldots, z_{n m_{n}}^{*}\right)$ among all the middle-level and bottom-level followers are uniquely determined respectively for the given value $\left(x^{*}, y_{i}^{*}\right)$ and $x^{*}$, which implies that the leader must optimize its objective over $I R$. According to the optimal solution set $O S$ in Definition 2.3(h), finding an optimal solution to model (1) is equivalent to solving the following problem:

$$
\min \left\{f^{(1)}\left(x, y_{1}, \ldots, y_{n}, z_{11}, \ldots, z_{1 m_{1}}, \ldots, z_{n 1}, \ldots, z_{n m_{n}}\right):\left(x, y_{1}, \ldots, y_{n}, z_{11}, \ldots, z_{1 m_{1}}, \ldots, z_{n 1}, \ldots, z_{n m_{n}}\right) \in I R\right\} .
$$

Therefore, problem (2) consists of minimizing a continuous function over a nonempty and compact set $I R$, which implies that there exists an optimal solution to the problem (2) that is also a Stackelberg-Nash solution to the MFTL decision model (1).

It is noticeable from the Theorem 2.1 that if the followers have multiple optimal solutions to respond to the parameter value $x$ of the leader, it will be difficult for the leader to realize its objective function value prior to the determination of the optimal solution taken by the followers [19]. In this case, if the followers cannot select the solution preferred by the leader, the leader may achieve its optimal solution outside $I R$, which implies that the MFTL decision model (1) may not have an optimal solution. Therefore, to avoid this situation in the presentation of solution algorithms, the assumption (3) is necessary.

Theorem 2.2 The IR can be expressed equivalently as a piecewise linear equality constraint comprised of supporting hyperplanes of $S$.

Proof. First, define

$$
\begin{array}{r}
F_{i j}\left(x, y_{i}, z_{i 1}, \ldots, z_{i(j-1)}, z_{i(j+1)}, \ldots, z_{i m_{i}}\right)=\min \left\{q_{i j j} z_{i j}: z_{i j} \in S_{i j}\left(x, y_{i}, z_{i 1}, \ldots, z_{i(j-1)}, z_{i(j+1)}, \ldots, z_{i m_{i}}\right)\right\}, \\
j=1,2, \ldots, m_{i}, i=1,2, \ldots, n . \\
F_{i}\left(x, y_{1}, \ldots, y_{i-1}, y_{i+1}, \ldots, y_{n}\right)=\min \left\{g_{i i} \hat{y}_{i}+\sum_{j=1}^{m_{i}} h_{i j} \hat{z}_{i j}:\left(\hat{y}_{i}, \hat{z}_{i 1}, \ldots, \hat{z}_{i m_{i}}\right) \in S_{i}\left(x, y_{1}, \ldots, y_{i-1}, y_{i+1}, \ldots, y_{n}\right),\right. \\
\left.q_{i j j} \hat{z}_{i j}=F_{i j}\left(x, \hat{y}_{i}, \hat{z}_{i 1}, \ldots, \hat{z}_{i(j-1)}, \hat{z}_{i(j+1)}, \ldots, \hat{z}_{i m_{i}}\right), j=1,2, \ldots, m_{i}\right\}, i=1,2, \ldots, n .
\end{array}
$$

Since $F_{i j}\left(x, y_{i}, z_{i 1}, \ldots, z_{i(j-1)}, z_{i(j+1)}, \ldots, z_{i m_{i}}\right)$ can be seen as a linear programming problem with parameters $x, y_{i}$ and $z_{i 1}, \ldots, z_{i(j-1)}, z_{i(j+1)}, \ldots, z_{i m_{i}}$, the dual problem of $F_{i j}\left(x, y_{i}, z_{i 1}, \ldots, z_{i(j-1)}, z_{i(j+1)}, \ldots, z_{i m_{i}}\right)$ can be written as:

$$
\max \left\{\left(A_{i j} x+P_{i j} y_{i}+\sum_{t=1, t \neq j}^{m_{i}} Q_{i j t} z_{i t}-b_{i j}\right) u_{i j}: Q_{i j} u_{i j} \geq-q_{i j j}, u_{i j} \geq 0\right\}, j=1,2, \ldots, m_{i}, i=1,2, \ldots, n .
$$

If both $F_{i j}\left(x, y_{i}, z_{i 1}, \ldots, z_{i(j-1)}, z_{i(j+1)}, \ldots, z_{i m_{i}}\right)$ and problem (3) have feasible solutions, according to the dual 
theorem of linear programming, both of them have optimal solutions and the same optimal objective function value. We know that a solution to problem (3) occurs at a vertex of its constraint region $U_{i j}=\left\{\left(u_{i j}: Q_{i j j} u_{i j} \geq-q_{i j j}, u_{i j} \geq 0\right\}\right.$. Adopting $u_{i j}^{1}, u_{i j}^{2}, \ldots, u_{i j}^{k_{i j}}$ to express all the vertices of $U_{i j}$, problem (3) can be written as:

$$
\max \left\{\left(A_{i j} x+P_{i j} y_{i}+\sum_{t=1, t \neq j}^{m_{i}} Q_{i j t} z_{i t}-b_{i j}\right) u_{i j}: u_{i j} \in\left\{u_{i j}^{1}, u_{i j}^{2}, \ldots, u_{i j}^{k_{i j}}\right\}, j=1,2, \ldots, m_{i}, i=1,2, \ldots, n .\right.
$$

Clearly, $F_{i j}\left(x, y_{i}, z_{i 1}, \ldots, z_{i(j-1)}, z_{i(j+1)}, \ldots, z_{i m_{i}}\right)$ is a piecewise linear function according to problem (4).

In the next step, we prove that $F_{i}\left(x, y_{1}, \ldots, y_{i-1}, y_{i+1}, \ldots, y_{n}\right)$ is also a piecewise linear function. Suppose that $\left(z_{i 1}^{1}, z_{i 2}^{1}, \ldots, z_{i m_{i}}^{1}\right), \ldots,\left(z_{i 1}^{s_{i}}, z_{i 2}^{s_{i}}, \ldots, z_{i m_{i}}^{s_{i}}\right)$ are solutions to the problem $\left\{F_{i j}\left(x, y_{i}, z_{i 1}, \ldots, z_{i(j-1)}, z_{i(j+1)}, \ldots, z_{i m_{i}}\right), j=1,2, \ldots, m_{i}\right\}$ for all $i=1,2, \ldots, n$. For each fixed $i$ and a solution $\left(z_{i 1}^{t_{i}}, z_{i 2}^{t_{i}}, \ldots, z_{i i_{i}}^{t_{i}}\right)$ where $t_{i}=1,2, \ldots, s_{i}$, $F_{i}\left(x, y_{1}, \ldots, y_{i-1}, y_{i+1}, \ldots, y_{n}\right)$ becomes a programming problem with parameters $x, y_{1}, \ldots, y_{i-1}, y_{i+1}, \ldots, y_{n}$ and $\left(z_{i 1}^{t_{i}}, z_{i 2}^{t_{i}}, \ldots, z_{i m_{i}}^{t_{i}}\right)$, and there are $s_{i}$ parameterized programming problems such as $\left.F_{i}\left(x, y_{1}, \ldots, y_{i-1}, y_{i+1}, \ldots, y_{n}\right)\right|_{\left(z_{i 1}^{1}, z_{i 2}^{1}, \ldots, z_{i m_{i}}^{1}\right)}, \ldots,\left.F_{i}\left(x, y_{1}, \ldots, y_{i-1}, y_{i+1}, \ldots, y_{n}\right)\right|_{\left(z_{i 1}^{s i}, z_{i 2}^{s i}, \ldots, z_{i m_{i}}^{s_{i}}\right)}$. Considering different combinations of $\left(z_{i 1}^{t_{i}}, z_{i 2}^{t_{i}}, \ldots, z_{i m_{i}}^{t_{i}}\right)$ for all $i=1,2, \ldots, n$, therefore, there are $\prod_{i=1}^{n} s_{i}$ parameterized programming problems $\left.\quad F_{i}\left(x, y_{1}, \ldots, y_{i-1}, y_{i+1}, \ldots, y_{n}\right)\right|_{\left(z_{i 1}^{t_{i}}, z_{i 2}^{t_{i}}, \ldots, z_{i m_{i}}^{t_{i}}\right)}$. Similarly, $\left.F_{i}\left(x, y_{1}, \ldots, y_{i-1}, y_{i+1}, \ldots, y_{n}\right)\right|_{\left(z_{i 1}^{t_{i}}, z_{i 2}^{t_{i}}, \ldots, z_{i m_{i}}^{t_{i}}\right)}$ is also a piecewise linear function as $F_{i j}\left(x, y_{i}, z_{i 1}, \ldots, z_{i(j-1)}, z_{i(j+1)}, \ldots, z_{i m_{i}}\right)$. Lastly, according to the above definition of $F_{i}\left(x, y_{1}, \ldots, y_{i-1}, y_{i+1}, \ldots, y_{n}\right)$, the inducible region $(I R)$ can be rewritten as:

$$
\begin{aligned}
& I R=\left\{\left(x, y_{1}, \ldots, y_{n}, z_{11}^{t_{1}}, \ldots, z_{1 m_{1}}^{t_{1}}, \ldots, z_{n 1}^{t_{n}}, \ldots, z_{n m_{n}}^{t_{n}}\right) \in S:\right. \\
& \left.g_{i i} y_{i}+\sum_{j=1}^{m_{i}} h_{i j} z_{i j}^{t_{i}}=\left.F_{i}\left(x, y_{1}, \ldots, y_{i-1}, y_{i+1}, \ldots, y_{n}\right)\right|_{\left(z_{i i}^{t}, z_{i}^{t_{i}}, \ldots, z_{i i_{i}}^{t_{i}}\right)} t_{i}=1,2, \ldots, s_{i}, i=1,2, \ldots, n\right\} \\
& =\left\{\left(x, y_{1}, \ldots, y_{n}, z_{11}^{t_{1}}, \ldots, z_{1 m_{1}}^{t_{1}}, \ldots, z_{n 1}^{t_{n}}, \ldots, z_{n m_{n}}^{t_{n}}\right) \in S:\right. \\
& \left.g_{i i} y_{i}=\left.F_{i}\left(x, y_{1}, \ldots, y_{i-1}, y_{i+1}, \ldots, y_{n}\right)\right|_{\left(z_{i 1}^{t_{i}}, z_{i}^{t_{i}}, \ldots, z_{i i_{i}}^{t_{i}}\right)}-\sum_{j=1}^{m_{i}} h_{i j} z_{i j}^{t_{i}}=0, t_{i}=1,2, \ldots, s_{i}, i=1,2, \ldots, n\right\},
\end{aligned}
$$

and it can be seen as a piecewise linear equality constraint for problem (2).

Corollary 2.1 The MFTL decision model (1) is equivalent to optimizing $f^{(1)}$ over a feasible region comprised of a piecewise linear equality constraint.

Corollary 2.2 An optimal solution to the MFTL decision model (1) occurs at a vertex of IR.

Proof. According to the equivalent form (2) of the MFTL decision model (1) and since $f^{(1)}\left(x, y_{1}, \ldots, y_{n}, z_{11}, \ldots, z_{1 m_{1}}, \ldots, z_{n 1}, \ldots, z_{n m_{n}}\right)$ is linear, an optimal solution to the problem must occur at a vertex of $I R$ if it exists.

Theorem 2.3 An optimal solution $\left(x^{*}, y_{1}^{*}, \ldots, y_{n}^{*}, z_{11}^{*}, \ldots, z_{1 m_{1}}^{*}, \ldots, z_{n 1}^{*}, \ldots, z_{n m_{n}}^{*}\right)$ to the MFTL decision model (1) 
occurs at a vertex of $S$.

Proof. Let $\left(x^{1}, y_{1}^{1}, \ldots, y_{n}^{1}, z_{11}^{1}, \ldots, z_{1 m_{1}}^{1}, \ldots, z_{n 1}^{1}, \ldots, z_{n m_{n}}^{1}\right), \ldots,\left(x^{t}, y_{1}^{t}, \ldots, y_{n}^{t}, z_{11}^{t}, \ldots, z_{1 m_{1}}^{t}, \ldots, z_{n 1}^{t}, \ldots, z_{n m_{n}}^{t}\right)$ express the distinct vertices of $S$. Since any point in $S$ can be written as a convex combination of these vertices, we can obtain $\left(x^{*}, y_{1}^{*}, \ldots, y_{n}^{*}, z_{11}^{*}, \ldots, z_{1 m_{1}}^{*}, \ldots, z_{n 1}^{*}, \ldots, z_{n m_{n}}^{*}\right)=\sum_{r=1}^{\bar{t}} \delta_{r}\left(x^{r}, y_{1}^{r}, \ldots, y_{n}^{r}, z_{11}^{r}, \ldots, z_{1 m_{1}}^{r}, \ldots, z_{n 1}^{r}, \ldots, z_{n m_{n}}^{r}\right) \quad$ where $\sum_{r=1}^{\bar{t}} \delta_{r}=1, \delta_{r}>0$, $r=1,2, \ldots, \bar{t}$ and $\bar{t} \leq t$. By the convexity of $F_{i}\left(x^{*}, y_{1}^{*}, \ldots, y_{i-1}^{*}, y_{i+1}^{*}, \ldots, y_{n}^{*}\right)$, let us write the constraints of model (1) in the piecewise linear form (5) discussed in Theorem 2.2:

$$
\begin{aligned}
0 & =\left.F_{i}\left(x^{*}, y_{1}^{*}, \ldots, y_{i-1}^{*}, y_{i+1}^{*}, \ldots, y_{n}^{*}\right)\right|_{\left(z_{i 1}^{*}, z_{i 2}^{*}, \ldots, z_{i m_{i}}^{*}\right)}-g_{i i} y_{i}^{*}-\sum_{j=1}^{m_{i}} h_{i j} z_{i j}^{*} \\
& \left.=\left.F_{i}\left(\sum_{r=1}^{\bar{t}} \delta_{r}\left(x^{r}, y_{1}^{r}, \ldots, y_{i-1}^{r}, y_{i+1}^{r}, \ldots, y_{n}^{r}\right)\right)\right|_{\left(z_{i 1}^{*}, z_{i 2}^{*}, \ldots, z_{i m_{i}}^{*}\right)}-g_{i i} \sum_{r=1}^{\bar{t}} \delta_{r} y_{i}^{r}-\sum_{j=1}^{m_{i}} h_{i j} \sum_{r=1}^{\bar{t}} \delta_{r} z_{i j}^{r}\right) \\
& \leq\left.\sum_{r=1}^{\bar{t}} \delta_{r} F_{i}\left(x^{r}, y_{1}^{r}, \ldots, y_{i-1}^{r}, y_{i+1}^{r}, \ldots, y_{n}^{r}\right)\right|_{\left(z_{i 1}^{*}, z_{i 2}^{*}, \ldots, z_{\left.i i_{i}\right)}^{*}\right)}-\sum_{r=1}^{\bar{t}} \delta_{r} g_{i i} y_{i}^{r}-\sum_{r=1}^{\bar{t}} \delta_{r}\left(\sum_{j=1}^{m_{i}} h_{i j} z_{i j}^{r}\right) \\
& =\sum_{r=1}^{\bar{t}} \delta_{r}\left(\left.F_{i}\left(x^{r}, y_{1}^{r}, \ldots, y_{i-1}^{r}, y_{i+1}^{r}, \ldots, y_{n}^{r}\right)\right|_{\left(z_{i 1}^{*}, z_{i 2}^{*}, \ldots, z_{i m_{i}}^{*}\right)}-g_{i i} y_{i}^{r}-\sum_{j=1}^{m_{i}} h_{i j} z_{i j}^{r}\right), i=1,2, \ldots, n .
\end{aligned}
$$

By the definition of $\left.F_{i}\left(x, y_{1}, \ldots, y_{i-1}, y_{i+1}, \ldots, y_{n}\right)\right|_{\left(z_{i 1}^{s_{i}}, z_{i 2}^{s_{i}}, \ldots, z_{i m_{i}}^{s_{i}}\right)}$, we have $\left.F_{i}\left(x^{r}, y_{1}^{r}, \ldots, y_{i-1}^{r}, y_{i+1}^{r}, \ldots, y_{n}^{r}\right)\right|_{\left(z_{i 1}^{*}, z_{i 2}^{*}, \ldots, z_{i m_{i}}^{*}\right)}=\min \left(g_{i i} y_{i}+\sum_{j=1}^{m_{i}} h_{i j} z_{i j}^{t_{i}}\right) \leq g_{i i} y_{i}^{r}+\sum_{j=1}^{m_{i}} h_{i j} z_{i j}^{r}, r=1,2, \ldots, \bar{t}, i=1,2, \ldots, n$. Thus, $\left.\quad F_{i}\left(x^{r}, y_{1}^{r}, \ldots, y_{i-1}^{r}, y_{i+1}^{r}, \ldots, y_{n}^{r}\right)\right|_{\left(z_{i 1}^{*}, z_{i 2}^{*}, \ldots, z_{i i_{i}}^{*}\right)}-g_{i i} y_{i}^{r}-\sum_{j=1}^{m_{i}} h_{i j} z_{i j}^{r} \leq 0, r=1,2, \ldots, \bar{t}, i=1,2, \ldots, n . \quad$ Because $\quad$ the preceding expression (6) must be held with $\delta_{r}>0, r=1,2, \ldots, \bar{t}$, there must exist $\left.F_{i}\left(x^{r}, y_{1}^{r}, \ldots, y_{i-1}^{r}, y_{i+1}^{r}, \ldots, y_{n}^{r}\right)\right|_{\left(z_{i 1}^{*}, z_{i 2}^{*}, \ldots, z_{\left.i i_{i}\right)}^{*}\right)}-g_{i i} y_{i}^{r}-\sum_{j=1}^{m_{i}} h_{i j} z_{i j}^{r}=0, r=1,2, \ldots, \bar{t}, i=1,2, \ldots, n$. These statements imply that $\left(x^{r}, y_{1}^{r}, \ldots, y_{n}^{r}, z_{11}^{r}, \ldots, z_{1 m_{1}}^{r}, \ldots, z_{n 1}^{r}, \ldots, z_{n m_{n}}^{r}\right) \in I R, r=1,2, \ldots, \bar{t}$, and that $\left(x^{*}, y_{1}^{*}, \ldots, y_{n}^{*}, z_{11}^{*}, \ldots, z_{1 m_{1}}^{*}, \ldots, z_{n 1}^{*}, \ldots, z_{n m_{n}}^{*}\right)$ can be denoted as a convex combination of the points in the $I R$. Since $\left(x^{*}, y_{1}^{*}, \ldots, y_{n}^{*}, z_{11}^{*}, \ldots, z_{1 m_{1}}^{*}, \ldots, z_{n 1}^{*}, \ldots, z_{n m_{n}}^{*}\right)$ is a vertex of the $I R$ according to Corollary 2.2, there must exist $\bar{t}=1$, which implies that $\left(x^{*}, y_{1}^{*}, \ldots, y_{n}^{*}, z_{11}^{*}, \ldots, z_{1 m_{1}}^{*}, \ldots, z_{n 1}^{*}, \ldots, z_{n m_{n}}^{*}\right)$ is a vertex of $S$.

Corollary 2.3 If $\left(x^{*}, y_{1}^{*}, \ldots, y_{n}^{*}, z_{11}^{*}, \ldots, z_{1 m_{1}}^{*}, \ldots, z_{n 1}^{*}, \ldots, z_{n m_{n}}^{*}\right)$ is a vertex of the IR, it is also a vertex of $S$.

\section{A MFTL Kth-Best algorithm}

Theorem 2.3 and Corollary 2.3 imply that we can find an optimal solution to the MFTL decision model (1) by enumerating vertices (also called extreme points) of the constraint region $S$, which clearly provide an appropriate way to develop the following MFTL Kth-Best algorithm to solve the problem. According to the notations and theoretical foundation respectively defined and demonstrated in Section 2, the main principle of the MFTL $K$ th-Best algorithm is proposed as follows.

To begin, consider the following linear programming problem:

$$
\min \left\{c x+\sum_{i=1}^{n} d_{i} y_{i}+\sum_{i=1}^{n} \sum_{j=1}^{m_{i}} e_{i j} z_{i j}:\left(x, y_{1}, \ldots, y_{n}, z_{11}, \ldots, z_{1 m_{1}}, \ldots, z_{n 1}, \ldots, z_{n m_{n}}\right) \in S\right\} .
$$


Let the vertices $\left(x^{1}, y_{1}^{1}, \ldots, y_{n}^{1}, z_{11}^{1}, \ldots, z_{1 m_{1}}^{1}, \ldots, z_{n 1}^{1}, \ldots, z_{n m_{n}}^{1}\right), \ldots,\left(x^{N}, y_{1}^{N}, \ldots, y_{n}^{N}, z_{11}^{N}, \ldots, z_{1 m_{1}}^{N}, \ldots, z_{n 1}^{N}, \ldots, z_{n m_{n}}^{N}\right) \quad$ of $\quad$ the constraint region $S$ denote the $N$-ranked basic feasible solutions to problem (7), such that $c x^{k}+\sum_{i=1}^{n} d_{i} y_{i}^{k}+\sum_{i=1}^{n} \sum_{j=1}^{m_{i}} e_{i j} z_{i j}^{k} \leq c x^{k+1}+\sum_{i=1}^{n} d_{i} y_{i}^{k+1}+\sum_{i=1}^{n} \sum_{j=1}^{m_{i}} e_{i j} z_{i j}^{k+1}, k=1,2, \ldots, N-1$. Solving the equivalent problem (2) of model (1) is then equivalent to finding the index $K^{*}=\min \left\{k \in\{1,2, \ldots, N\}:\left(x^{k}, y_{1}^{k}, \ldots, y_{n}^{k}, z_{11}^{k}, \ldots, z_{1 m_{1}}^{k}, \ldots\right.\right.$, $\left.\left.z_{n 1}^{k}, \ldots, z_{n m_{n}}^{k}\right) \in I R\right\}$, which ensures that $\left(x^{K^{*}}, y_{1}^{K^{*}}, \ldots, y_{n}^{K^{*}}, z_{11}^{K^{*}}, \ldots, z_{1 m_{1}}^{K^{*}}, \ldots, z_{n 1}^{K^{*}}, \ldots, z_{n m_{n}}^{K^{*}}\right)$ is an optimal solution to model (1). Therefore, we need to verify whether or not $\left(x^{K^{*}}, y_{1}^{K^{*}}, \ldots, y_{n}^{K^{*}}, z_{11}^{K^{*}}, \ldots, z_{1 m_{1}}^{K^{*}}, \ldots, z_{n 1}^{K^{*}}, \ldots, z_{n m_{n}}^{K^{*}}\right) \in I R$ under the condition $\left(x^{K^{*}}, y_{1}^{K^{*}}, \ldots, y_{n}^{K^{*}}, z_{11}^{K^{*}}, \ldots, z_{1 m_{1}}^{K^{*}}, \ldots, z_{n 1}^{K^{*}}, \ldots, z_{n m_{n}}^{K^{*}}\right) \in S$. If $\left(y_{i}^{K^{*}}, z_{i 1}^{K^{*}}, \ldots, z_{i m_{i}}^{K^{*}}\right) \in P_{i}\left(x^{K^{*}}, y_{1}^{K^{*}}, \ldots\right.$, $\left.y_{i-1}^{K^{*}}, y_{i+1}^{K^{*}}, \ldots, y_{n}^{K^{*}}\right)$ for all $i=1,2, \ldots, n$ that means $\left(y_{i}^{K^{*}}, z_{i 1}^{K^{*}}, \ldots, z_{i m_{i}}^{K^{*}}\right)$ is an optimal solution to the problem (1c-1f) under the fixed $x=x^{K^{*}}, y_{1}=y_{1}^{K^{*}}, \ldots, y_{i-1}=y_{i-1}^{K^{*}}, y_{i+1}=y_{i+1}^{K^{*}}, \ldots, y_{n}=y_{n}^{K^{*}}$ for $i=1,2, \ldots, n$, there exists $\left(y_{1}^{K^{*}}, \ldots, y_{n}^{K^{*}}, z_{11}^{K^{*}}, \ldots, z_{1 m_{1}}^{K^{*}}, \ldots, z_{n 1}^{K^{*}}, \ldots, z_{n m_{n}}^{K^{*}}\right) \in P\left(x^{K^{*}}\right) \quad, \quad$ thus, $\quad\left(x^{K^{*}}, y_{1}^{K^{*}}, \ldots, y_{n}^{K^{*}}, z_{11}^{K^{*}}, \ldots, z_{1 m_{1}}^{K^{*}}, \ldots, z_{n 1}^{K^{*}}, \ldots, z_{n m_{n}}^{K_{n}}\right) \in I R \quad$ by Definition 2.3(g). As this requires finding the $K^{*}$ th best vertex of $S$ to obtain an optimal solution to model (1), the algorithm is named the MFTL Kth-Best algorithm.

Second, we need to verify whether or not $\left(y_{i}^{K^{*}}, z_{i 1}^{K^{*}}, \ldots, z_{i m_{i}}^{K^{*}}\right) \in P_{i}\left(x^{K^{*}}, y_{1}^{K^{*}}, \ldots, y_{i-1}^{K^{*}}, y_{i+1}^{K^{*}}, \ldots, y_{n}^{K^{*}}\right)$ through solving problem (1c-1f). For $i=1,2, \ldots, n$ and fixing the given $x=x^{K^{*}}, y_{1}=y_{1}^{K^{*}}, \ldots, y_{i-1}=y_{i-1}^{K^{*}}$, $y_{i+1}=y_{i+1}^{K^{*}}, \ldots, y_{n}=y_{n}^{K^{*}},(1 \mathrm{c}-1 \mathrm{f})$ can be seen as the problem (8) by Definition 2.3(f):

$$
\min \left\{c_{i} x+\sum_{s=1}^{n} g_{i s} y_{s}+\sum_{j=1}^{m_{i}} h_{i j} z_{i j}:\left(y_{i}, z_{i 1}, \ldots, z_{i m_{i}}\right) \in P_{i}\left(x, y_{1}, \ldots, y_{i-1}, y_{i+1}, \ldots, y_{n}\right)\right\} .
$$

For the given $x=x^{K^{*}}, y_{1}=y_{1}^{K^{*}}, \ldots, y_{i-1}=y_{i-1}^{K^{*}}, \quad y_{i+1}=y_{i+1}^{K^{*}}, \ldots, y_{n}=y_{n}^{K^{*}}$, consider the following linear programming problem (9):

$$
\min \left\{c_{i} x+\sum_{s=1}^{n} g_{i s} y_{s}+\sum_{j=1}^{m_{i}} h_{i j} z_{i j}:\left(y_{i}, z_{i 1}, \ldots, z_{i m_{i}}\right) \in S_{i}\left(x, y_{1}, \ldots, y_{i-1}, y_{i+1}, \ldots, y_{n}\right)\right\}
$$

and the vertices $\left(y_{i}^{1}, z_{i 1}^{1}, \ldots, z_{i m_{i}}^{1}\right), \ldots,\left(y_{i}^{N_{i}}, z_{i 1}^{N_{i}}, \ldots, z_{i m_{i}}^{N_{i}}\right)$ of $S_{i}\left(x, y_{1}, \ldots, y_{i-1}, y_{i+1}, \ldots, y_{n}\right)$ become the ranked basic feasible solutions to problem (9), such that $c_{i} x^{K^{*}}+\sum_{\substack{s=1, s \neq i}}^{n} g_{i s} y_{s}^{K^{*}}+g_{i i} y_{i}^{k_{i}}+\sum_{j=1}^{m_{i}} h_{i j} z_{i j}^{k_{i}} \leq$ $c_{i} x^{K^{*}}+\sum_{\substack{s=1, s \neq i}}^{n} g_{i s} y_{s}^{K^{*}}+g_{i i} y_{i}^{k_{i}+1}+\sum_{j=1}^{m_{i}} h_{i j} z_{i j}^{k_{i j}+1}, k_{i}=1,2, \ldots, N_{i}-1, i=1,2, \ldots, n$. Solving problem (8) is then equivalent to finding the index $K_{i}^{*}=\min \left\{k_{i} \in\left\{1,2, \ldots, N_{i}\right\}:\left(y_{i}^{k_{i}}, z_{i 1}^{k_{i}}, \ldots, z_{i m_{i}}^{k_{i}}\right) \in P_{i}\left(x^{K^{*}}, y_{1}^{K^{*}}, \ldots, y_{i-1}^{K^{*}}, y_{i+1}^{K^{*}}, \ldots, y_{n}^{K^{*}}\right)\right\}$, which ensures that $\left(y_{1}^{K_{i}^{*}}, z_{i 1}^{K_{i}^{*}}, \ldots, z_{i m_{i}}^{K_{i}^{*}}\right)$ is an optimal solution to (1c-1f) where $i=1,2, \ldots, n$. If $\left(y_{1}^{K_{i}^{*}}, z_{i 1}^{K_{i}^{*}}, \ldots, z_{i m_{i}}^{K_{i}^{*}}\right)=\left(y_{i}^{K^{*}}, z_{i 1}^{K^{*}}, \ldots, z_{i m_{i}}^{K^{*}}\right)$, we can conclude that $\left(y_{i}^{K^{*}}, z_{i 1}^{K^{*}}, \ldots, z_{i m_{i}}^{K^{*}}\right) \in P_{i}\left(x^{K^{*}}, y_{1}^{K^{*}}, \ldots, y_{i-1}^{K^{*}}, y_{i+1}^{K^{*}}, \ldots, y_{n}^{K^{*}}\right)$.

Before the detailed procedures of the MFTL Kth-Best algorithm are presented, the notations and indexes used in the algorithm are explained in Table 1. 
Table 1 Notations and indexes used in the MFTL $K$ th-Best algorithm

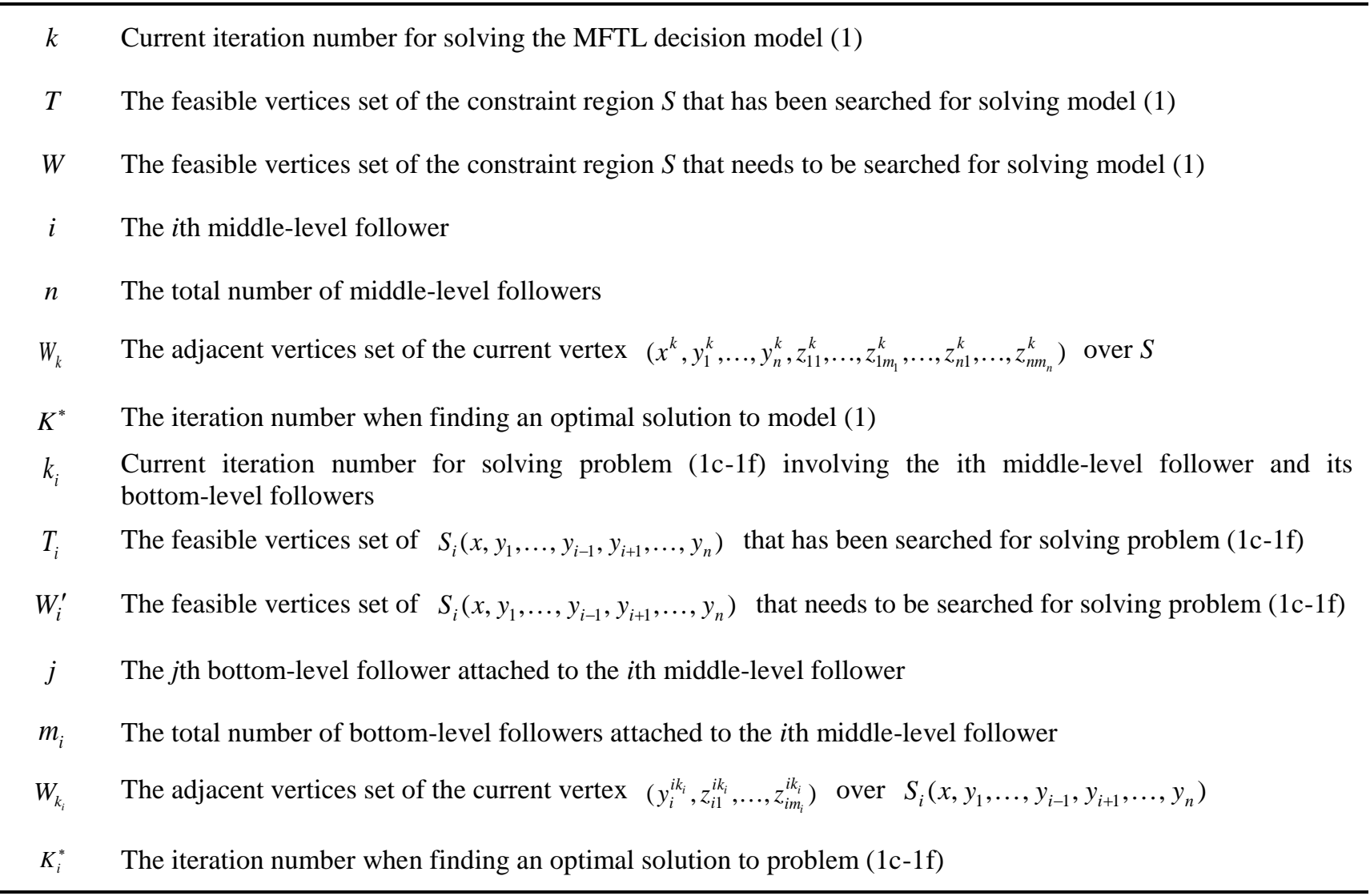

The MFTL Kth-Best algorithm: The input is the coefficients of model (1), and the output is an optimal solution to model (1) and the iteration number $K^{*}$.

[Begin]

Step 1: Set $k=1$, adopt the simplex method to obtain an optimal solution $\left(x^{1}, y_{1}^{1}, \ldots, y_{n}^{1}, z_{11}^{1}, \ldots, z_{1 m_{1}}^{1}, \ldots, z_{n 1}^{1}, \ldots, z_{n m_{n}}^{1}\right)$ to the linear programming problem (7). Let $T=\varnothing$ and $W=\left\{\left(x^{1}, y_{1}^{1}, \ldots, y_{n}^{1}, z_{11}^{1}, \ldots, z_{1 m_{1}}^{1}, \ldots, z_{n 1}^{1}, \ldots, z_{n m_{n}}^{1}\right)\right\}$. Set $i=1$ and go to Step 2 .

Step 2: Put $x=x^{k}, y_{1}=y_{1}^{k}, \ldots, y_{i-1}=y_{i-1}^{k}, y_{i+1}=y_{i+1}^{k}, \ldots, y_{n}=y_{n}^{k}$, solve the problem (1c-1f) or problem (8) and obtain an optimal solution $\left(\hat{y}_{i}, \hat{z}_{i 1}, \ldots, \hat{z}_{i m_{i}}\right)$ using the following subroutine Step 2.1-Step 2.5. Then go to Step 3.

Step 2.1: Set $x=x^{k}$ and $k_{i}=1$, adopt the simplex method to obtain the optimal solution $\left(y_{i}^{i 1}, z_{i 1}^{i 1}, \ldots, z_{i m_{i}}^{i 1}\right)$ to the linear programming problem (9). Within the subroutine, let $T_{i}=\varnothing$ and $W_{i}^{\prime}=\left\{\left(y_{1}^{i 1}, z_{i 1}^{i 1}, \ldots, z_{i m_{i}}^{i 1}\right)\right\}$. Set $j=1$ and go to Step 2.2 .

Step 2.2: Put $x=x^{k}, y_{i}=y_{i}^{i k_{i}}, z_{i 1}=z_{i 1}^{i k_{i}}, \ldots, z_{i(j-1)}=z_{i(j-1)}^{i k_{i}}, z_{i(j+1)}=z_{i(j+1)}^{i k_{i}}, \ldots, z_{i m_{i}}=z_{i m_{i}}^{i k_{i}}$ and adopt the simplex method to solve the problem (10):

$$
\min \left\{c_{i j} x+p_{i j} y_{i}+\sum_{t=1}^{m_{i}} q_{i j t} z_{i t}: z_{i j} \in S_{i j}\left(x, y_{i}, z_{i 1}, \ldots, z_{i(j-1)}, z_{i(j+1)}, \ldots, z_{i m_{i}}\right)\right\}
$$


and obtain the optimal solution $\tilde{z}_{i j}$.

Step 2.3: If $\tilde{z}_{i j} \neq z_{i j}^{i k_{i}}$, go to Step 2.4. If $\tilde{z}_{i j}=z_{i j}^{i k_{i}}$ and $j \neq m_{i}$, set $j=j+1$ and go to Step 2.2. If $\tilde{z}_{i j}=z_{i j}^{i k_{i}}$ and $j=m_{i}$, stop the subroutine, $K_{i}^{*}=k_{i}$ and go to Step 2 with $\left(\hat{y}_{i}, \hat{z}_{i 1}, \ldots, \hat{z}_{i m_{i}}\right)=\left(y_{i}^{i k_{i}}, z_{i 1}^{i k_{i}}, \ldots, z_{i m_{i}}^{i k_{i}}\right)$.

Step 2.4: Let $W_{k_{i}}$ denote the set of adjacent vertices of $\left(y_{i}^{i k_{i}}, z_{i 1}^{i k_{i}}, \ldots, z_{i m_{i}}^{i k_{i}}\right)$ that $\left(y_{i}, z_{i 1}, \ldots, z_{i m_{i}}\right) \in W_{k_{i}}$ implies $\quad c_{i} x^{k}+\sum_{\substack{s=1, s \neq i}}^{n} g_{i s} y_{s}^{k}+g_{i i} y_{i}+\sum_{j=1}^{m_{i}} h_{i j} z_{i j} \geq c_{i} x^{k}+\sum_{\substack{s=1, s \neq i}}^{n} g_{i s} y_{s}^{k}+g_{i i} y_{i}^{i k_{i}}+\sum_{j=1}^{m_{i}} h_{i j} z_{i j}^{i k_{i}} \quad \cdot \quad$ Let $T_{i}=T_{i} \bigcup\left\{\left(y_{i}^{i k_{i}}, z_{i 1}^{i k_{i}}, \ldots, z_{i m_{i}}^{i k_{i}}\right)\right\}$ and $W_{i}^{\prime}=\left(W_{i}^{\prime} \bigcup W_{k_{i}}\right) \backslash T_{i}$. Go to Step 2.5.

Step 2.5: Set $k_{i}=k_{i}+1$ and choose $\left(y_{i}^{i k_{i}}, z_{i 1}^{i k_{i}}, \ldots, z_{i m_{i}}^{i k_{i}}\right)$ such that $c_{i} x^{k}+\sum_{\substack{s=1, s \neq i}}^{n} g_{i s} y_{s}^{k}+g_{i i} y_{i}^{i k_{i}}+\sum_{j=1}^{m_{i}} h_{i j} z_{i j}^{i k_{i}}$ $=\min \left\{c_{i} x^{k}+\sum_{\substack{s=1, s \neq i}}^{n} g_{i s} y_{s}^{k}+g_{i i} y_{i}+\sum_{j=1}^{m_{i}} h_{i j} z_{i j}:\left(y_{i}, z_{i 1}, \ldots, z_{i m_{i}}\right) \in W_{i}^{\prime}\right\}$. Set $j=1$ and go to Step 2.2.

Step 3: If $\left(\hat{y}_{i}, \hat{z}_{i 1}, \ldots, \hat{z}_{i m_{i}}\right) \neq\left(y_{i}^{k}, z_{i 1}^{k}, \ldots, z_{i m_{i}}^{k}\right)$, go to Step 4. If $\left(\hat{y}_{i}, \hat{z}_{i 1}, \ldots, \hat{z}_{i m_{i}}\right)=\left(y_{i}^{k}, z_{i 1}^{k}, \ldots, z_{i m_{i}}^{k}\right)$ and $i \neq n$, set $i=i+1$ and go to Step 2. If $\left(\hat{y}_{i}, \hat{z}_{i 1}, \ldots, \hat{z}_{i m_{i}}\right)=\left(y_{i}^{k}, z_{i 1}^{k}, \ldots, z_{i m_{i}}^{k}\right)$ and $i=n$, stop and $\left(x^{k}, y_{1}^{k}, \ldots, y_{n}^{k}, z_{11}^{k}, \ldots, z_{1 m_{1}}^{k}, \ldots, z_{n 1}^{k}, \ldots, z_{n m_{n}}^{k}\right)$ is an optimal solution to the MFTL decision model (1) and $K^{*}=k$.

Step 4: Let $W_{k}$ denote the set of adjacent vertices of $\left(x^{k}, y_{1}^{k}, \ldots, y_{n}^{k}, z_{11}^{k}, \ldots, z_{1 m_{1}}^{k}, \ldots, z_{n 1}^{k}, \ldots, z_{n m_{n}}^{k}\right)$ such that $\left(x, y_{1}, \ldots, y_{n}, z_{11}, \ldots, z_{1 m_{1}}, \ldots, z_{n 1}, \ldots, z_{n m_{n}}\right) \in W_{k}$ implies $c x+\sum_{i=1}^{n} d_{i} y_{i}+\sum_{i=1}^{n} \sum_{j=1}^{m_{i}} e_{i j} z_{i j} \geq c x^{k}+\sum_{i=1}^{n} d_{i} y_{i}^{k}+\sum_{i=1}^{n} \sum_{j=1}^{m_{i}} e_{i j} z_{i j}^{k}$. Let $T=T \bigcup\left\{\left(x^{k}, y_{1}^{k}, \ldots, y_{n}^{k}, z_{11}^{k}, \ldots, z_{1 m_{1}}^{k}, \ldots, z_{n 1}^{k}, \ldots, z_{n m_{n}}^{k}\right)\right\}$ and $W=\left(W \bigcup W_{k}\right) \backslash T$. Go to Step 5 .

Step 5: Set $k=k+1$ and choose $\left(x^{k}, y_{1}^{k}, \ldots, y_{n}^{k}, z_{11}^{k}, \ldots, z_{1 m_{1}}^{k}, \ldots, z_{n 1}^{k}, \ldots, z_{n m_{n}}^{k}\right)$ such that $c x^{k}+\sum_{i=1}^{n} d_{i} y_{i}^{k}+\sum_{i=1}^{n} \sum_{j=1}^{m_{i}} e_{i j} z_{i j}^{k}$ $=\min \left\{c x+\sum_{i=1}^{n} d_{i} y_{i}+\sum_{i=1}^{n} \sum_{j=1}^{m_{i}} e_{i j} z_{i j}:\left(x, y_{1}, \ldots, y_{n}, z_{11}, \ldots, z_{1 m_{1}}, \ldots, z_{n 1}, \ldots, z_{n m_{n}}\right) \in W\right\}$. Set $i=1$ and go to Step 2.

[End]

Within the MFTL Kth-Best algorithm, Step 2 and its subroutine (Step 2.1-2.5) are adopted to obtain an optimal solution to problem (1c-1f) of the ith middle-level follower and its bottom-level followers under the given decision $\left(x, y_{1}, \ldots, y_{i-1}, y_{i+1}, \ldots, y_{n}\right)$ from the leader and other middle-level followers. Step 3 is repeatedly performed to see whether or not the current vertex is an element involved in the $I R$. If the current vertex occurs outside $I R$, the algorithm will go to Step 4 in which the adjacent vertices of the current vertex will be found and added to the vertices set $W$ that needs to be searched. Step 5 is developed to choose a vertex from the vertices set $W$ to optimize the objective function of problem (7) and prepare for the next iteration to verify whether or not the vertex is an element of the $I R$. 
We then use a simple numerical example, shown as the following Example 1, to illustrate how the MFTL Kth-Best algorithm works. Also, we will illustrate the algorithm through a case study in Section 5.

Example 1 We assume that the example involves one leader, two middle-level followers and two bottom-level followers attached to each middle-level follower, which means that $n=2, m_{1}=m_{2}=2$ in model (1). For $X=\{x: x \geq 0\}, Y_{i}=\left\{y_{i}: y_{i} \geq 0\right\}, Z_{i j}=\left\{z_{i j}: z_{i j} \geq 0\right\}, j=1,2, i=1,2$, coefficients of the decision variables in model (1) are shown in Table 2.

Table 2 Coefficients of model (1) in Example 1

\begin{tabular}{|c|c|c|}
\hline \multirow{2}{*}{ Decision entity } & Coefficients of model (1) & \multirow[b]{2}{*}{ Coefficients of constraint conditions } \\
\hline & Coefficients of objective functions & \\
\hline Leader & $\begin{array}{l}c=-1, d_{1}=1, d_{2}=-2, e_{11}=3 \\
e_{12}=1, e_{21}=-1, e_{22}=-1\end{array}$ & $\begin{array}{l}A=(1,1)^{T}, B_{1}=(1,0)^{T}, B_{2}=(1,0)^{T}, C_{11}=(1,0)^{T}, \\
C_{12}=(2,0)^{T}, C_{21}=(2,0)^{T}, C_{22}=(1,0)^{T}, b=(14,1.5)^{T}\end{array}$ \\
\hline Follower 1 & $c_{1}=1, g_{11}=-1, g_{12}=1, h_{11}=1, h_{12}=1$ & $\begin{array}{l}A_{1}=(2,0)^{T}, D_{11}=(1,1)^{T}, D_{12}=(1,0)^{T}, E_{11}=(1,0)^{T} \\
E_{12}=(1,0)^{T}, b_{1}=(8.5,1)^{T}\end{array}$ \\
\hline Follower 2 & $c_{2}=1, g_{21}=-1, g_{22}=2, h_{21}=1, h_{22}=1$ & $\begin{array}{l}A_{2}=(1,0)^{T}, D_{21}=(-1,-1)^{T}, D_{22}=(1,-1)^{T}, E_{21}=(1,0)^{T}, \\
E_{22}=(1,0)^{T}, b_{2}=(3,-1.5)^{T}\end{array}$ \\
\hline Follower 11 & $c_{11}=1, p_{11}=1, q_{111}=2, q_{112}=1$ & $\begin{array}{l}A_{11}=(-1,0)^{T}, P_{11}=(-1,0)^{T}, Q_{111}=(-1,1)^{T} \\
Q_{112}=(-2,0)^{T}, b_{11}=(-10,3)^{T}\end{array}$ \\
\hline Follower 12 & $c_{12}=2, p_{12}=1, q_{121}=1, q_{122}=1$ & $\begin{array}{l}A_{12}=(-1,1)^{T}, P_{12}=(-1,0)^{T}, Q_{121}=(-1,1)^{T} \\
Q_{122}=(-1,1)^{T}, b_{12}=(-7,6)^{T}\end{array}$ \\
\hline Follower 21 & $c_{21}=1, p_{21}=1, q_{211}=-3, q_{212}=1$ & $\begin{array}{l}A_{21}=(-1,0)^{T}, P_{21}=(-1,1)^{T}, Q_{211}=(-1,1)^{T} \\
Q_{212}=(-2,0)^{T}, b_{21}=(-5.5,1.5)^{T}\end{array}$ \\
\hline Follower 22 & $c_{22}=1, p_{22}=2, q_{221}=2, q_{222}=-1$ & $\begin{array}{l}A_{22}=(1,0)^{T}, P_{22}=(1,0)^{T}, Q_{221}=(1,1)^{T} \\
Q_{222}=(1,1)^{T}, b_{22}=(4,2.5)^{T}\end{array}$ \\
\hline
\end{tabular}

Detailed procedures of the MFTL Kth-Best algorithm that are executed to solve the example 1 are shown as follows.

\section{Iteration 1}

Step 1: Set $k=1$ and adopt the simplex method to obtain an optimal solution to the following linear programming problem (11) in the format (7):

$$
\min \left\{c x+\sum_{i=1}^{2} d_{i} y_{i}+\sum_{i=1}^{2} \sum_{j=1}^{2} e_{i j} z_{i j}:\left(x, y_{1}, y_{2}, z_{11}, z_{12}, z_{21}, z_{22}\right) \in S\right\} .
$$

The optimal solution to problem (12) is $\left(x^{1}, y_{1}^{1}, y_{2}^{1}, z_{11}^{1}, z_{12}^{1}, z_{21}^{1}, z_{22}^{1}\right)=(1,1,0.5,0.5,4.5,0,2)$ and now $T=\varnothing$, $W=\{(1,1,0.5,0.5,4.5,0,2)\}$. Set $i=1$ and go to Step 2 .

Step 2: Put $x=x^{1}=1, y_{2}=y_{2}^{1}=0.5$ and solve the problem (12) of the middle-level follower $i(=1)$ and its bottom-level followers in the format (8):

$$
\min \left\{c_{1} x+\sum_{s=1}^{2} g_{1 s} y_{s}+\sum_{j=1}^{2} h_{1 j} z_{1 j}:\left(y_{1}, z_{11}, z_{12}\right) \in P_{1}\left(x, y_{2}\right)\right\} .
$$

We can get an optimal solution $\left(\hat{y}_{1}, \hat{z}_{11}, \hat{z}_{12}\right)=(1,2,3)$ by Steps $2.1-2.5$ of the MFTL $K$ th-Best algorithm and go to Step 3. 
Step 3: $\left(\hat{y}_{1}, \hat{z}_{11}, \hat{z}_{12}\right) \neq\left(y_{1}^{1}, z_{11}^{1}, z_{12}^{1}\right)$ and go to Step 4 .

Step 4: Find the set $W_{1}$ of adjacent vertices of $\left(x^{1}, y_{1}^{1}, y_{2}^{1}, z_{11}^{1}, z_{12}^{1}, z_{21}^{1}, z_{22}^{1}\right)$ and now $W_{1}=\{(1,1,0.5,1,4,0,2.5)$, $(1,1,0.5,2,3,1,1.5),(1,1,0.5,2,3,0,2),(0,1,1.5,2.5,3.5,0,2),(0,1,0.5,2,4,0,2.5)\}, T=T \bigcup\{(1,1,0.5,0.5,4.5,0,2)\}=\{(1,1,0.5,0.5,4.5,0,2)\}$, $W=W \cup W_{1} \backslash T=W_{1}$. Go to Step 5.

\section{Iteration 2}

Step 5: Set $k=k+1=2$ and choose $\left(x^{2}, y_{1}^{2}, y_{2}^{2}, z_{11}^{2}, z_{12}^{2}, z_{21}^{2}, z_{22}^{2}\right)=(1,1,0.5,1,4,0,2.5)$ from the vertices set $W$ such that $f^{(1)}\left(x^{2}, y_{1}^{2}, y_{2}^{2}, z_{11}^{2}, z_{12}^{2}, z_{21}^{2}, z_{22}^{2}\right)=\min \left\{c x+\sum_{i=1}^{2} d_{i} y_{i}+\sum_{i=1}^{2} \sum_{j=1}^{2} e_{i j} z_{i j}:\left(x, y_{1}, y_{2}, z_{11}, z_{12}, z_{21}, z_{22}\right) \in W\right\}$, set $i=1$ and go to Step 2.

Step 2: Put $x=x^{2}=1, y_{2}=y_{2}^{2}=0.5$ and solve the problem (12). We can get an optimal solution $\left(\hat{y}_{1}, \hat{z}_{11}, \hat{z}_{12}\right)=(1,2,3) \quad$ by Steps $2.1-2.5$ of the MFTL $K$ th-Best algorithm and go to Step 3.

Step 3: $\left(\hat{y}_{1}, \hat{z}_{11}, \hat{z}_{12}\right) \neq\left(y_{1}^{2}, z_{11}^{2}, z_{12}^{2}\right)$ and go to Step 4 .

Step 4: Find the set $W_{2}$ of adjacent vertices of $\left(x^{2}, y_{1}^{2}, y_{2}^{2}, z_{11}^{2}, z_{12}^{2}, z_{21}^{2}, z_{22}^{2}\right)$ and now $W_{2}=\{(1,1,0.5,2,3,0,2.5),(0,1,1.5,3,3,0,2.5)\}, T=T \cup\{(1,1,0.5,1,4,0,2.5)\}=\{(1,1,0.5,0.5,4.5,0,2),(1,1,0.5,1,4,0,2.5)\}$, $W=W \cup W_{2} \backslash T=\{(1,1,0.5,2,3,1,1.5),(1,1,0.5,2,3,0,2),(0,1,1.5,2.5,3.5,0,2),(0,1,0.5,2,4,0,2.5),(1,1,0.5,2,3,0,2.5)$, $(0,1,1.5,3,3,0,2.5)\}$. Go to Step 5 .

\section{Iteration 3}

Step 5: Set $k=k+1=3$ and choose $\left(x^{3}, y_{1}^{3}, y_{2}^{3}, z_{11}^{3}, z_{12}^{3}, z_{21}^{3}, z_{22}^{3}\right)=(1,1,0.5,2,3,1,1.5)$ from the vertices set $W$ such that $f^{(1)}\left(x^{3}, y_{1}^{3}, y_{2}^{3}, z_{11}^{3}, z_{12}^{3}, z_{21}^{3}, z_{22}^{3}\right)=\min \left\{c x+\sum_{i=1}^{2} d_{i} y_{i}+\sum_{i=1}^{2} \sum_{j=1}^{2} e_{i j} z_{i j}:\left(x, y_{1}, y_{2}, z_{11}, z_{12}, z_{21}, z_{22}\right) \in W\right\}$, set $i=1$ and go to Step 2.

Step 2: Put $x=x^{3}=1, y_{2}=y_{2}^{3}=0.5$ and solve the problem (12). We can get an optimal solution $\left(\hat{y}_{1}, \hat{z}_{11}, \hat{z}_{12}\right)=(1,2,3)$ by Steps 2.1-2.5 of the MFTL Kth-Best algorithm and go to Step 3 .

Step 3: Clearly, $\left(\hat{y}_{1}, \hat{z}_{11}, \hat{z}_{12}\right)=\left(y_{1}^{3}, z_{11}^{3}, z_{12}^{3}\right)=(1,2,3) \quad$, and $i \neq n$, set $i=i+1=2$ and go to Step 2 .

Step 2: Put $x=x^{3}=1, y_{1}=y_{1}^{3}=1$ and solve the problem (13) of the middle-level follower $i(=2)$ and its bottom-level followers in the format (8):

$$
\min \left\{c_{2} x+\sum_{s=1}^{2} g_{2 s} y_{s}+\sum_{j=1}^{2} h_{2 j} z_{2 j}:\left(y_{1}, z_{21}, z_{22}\right) \in P_{2}\left(x, y_{1}\right)\right\} .
$$

We can get an optimal solution $\left(\hat{y}_{2}, \hat{z}_{21}, \hat{z}_{22}\right)=(0.5,1,1.5)$ by Steps $2.1-2.5$ of the MFTL $K$ th-Best algorithm and go to Step 3.

Step 3: $\left(\hat{y}_{2}, \hat{z}_{21}, \hat{z}_{22}\right)=\left(y_{2}^{3}, z_{21}^{3}, z_{22}^{3}\right)=(0.5,1,1.5)$ and $i=n=2$, stop and $\left(x^{3}, y_{1}^{3}, y_{2}^{3}, z_{11}^{3}, z_{12}^{3}, z_{21}^{3}, z_{22}^{3}\right)=(1,1,0.5,2,3,1,1.5)$ is an optimal solution to the example 1 and the iteration number $K^{*}=k=3$.

We finally find an optimal solution to the Example 1 through three iterations, which means that we enumerated three vertices to get an optimal solution. The objective function values of all decision entities are 
$f^{(1)}=5.5, f_{1}^{(2)}=5.5, f_{2}^{(2)}=3.5, f_{11}^{(3)}=9.0, f_{12}^{(3)}=8.0, f_{21}^{(3)}=0, f_{22}^{(3)}=2.5$. As we can see from the simple numerical example, the MFTL Kth-Best algorithm provides a convenient way to solve this kind of reference-uncooperative MFTL decision problem. However, there are still two practical issues in applying the proposed MFTL decision model and Kth-Best algorithm to deal with MFTL decision cases in the real world. One is that it is imprecise or ambiguous for decision entities to evaluate a solution whether or not decision entities desire this through their respective objective values; the other is that it remains difficult and inefficient to find an optimal manual solution if we have to search a mass of vertices using the MFTL $K$ th-Best algorithm. Therefore, it is becoming necessary and urgent to propose an approach to recognize the satisfactory degree of decision entities towards solutions, and develop a decision support system driven by the MFTL Kth-Best algorithm to assist decision makers in solving such MFTL decision problems accurately and efficiently. To overcome these issues, an evaluation approach of solutions using fuzzy programming will be proposed in Section 4. In Section 5, we will deal with a real-world reference-uncooperative MFTL decision problem by means of a tri-level decision support system driven by the proposed MFTL decision techniques.

\section{Evaluation of solutions}

We are able to find an optimal solution for the MFTL decision model (1) using the proposed MFTL $K$ th-Best algorithm based on related theoretical properties. However, it is difficult to illustrate the operations of the complex MFTL decision-making process by the optimal solution defined by Definition 2.3 because the solution only represents the decision result rather than the decision process. In this section, we will use a fuzzy programming approach to evaluate the solution obtained and illustrate why decision entities have to achieve and accept the final result during the MFTL decision-making process.

Within a MFTL decision-making process, each decision entity seeks to optimize its own objective but its decision is affected by actions of others, thus, decision entities achieve a compromised result with a possible relaxation rather than their individual best solutions as desired. Since it is imprecise or ambiguous for decision entities to identify a compromised result whether or not they desire it, the objective functions can be transformed into fuzzy goals using an imprecise aspiration level. We use $f^{\min }$ and $f^{\max }$ to denote the individual best and worst results respectively that a decision entity may achieve. Finally, the compromised objective value of the decision entity must be involved in the interval $\left[f^{\min }, f^{\max }\right]$. Therefore, we can elicit membership functions $\mu(f)$ to characterize fuzzy goals over the domain $\left[f^{\min }, f^{\max }\right]$ for the objective functions, which can also be adopted to describe the satisfactory degree of decision entities towards a solution or an objective value. For example, a decision entity specifies the objective value $f^{0}$ such that the satisfactory degree is 0 , that is $\mu\left(f^{0}\right)=0$, while the value $f^{1}$ of the objective function such that $\mu\left(f^{1}\right)=1$ means that the satisfactory degree is 1 . Clearly, if an objective value $f$ is undesired (larger) than $f^{0}$, it is defined that $\mu(f)=0$; whereas $\mu(f)=1$ if an objective value $f$ is desired (smaller) than $f^{1}$. In this study, for the sake of simplicity, we assume that $f^{0}$ and $f^{1}$ are specified as $f^{0}=f^{\max }$ and $f^{1}=f^{\min }$, 
and that the membership functions are linear versions shown as Fig. 2 although they do not always need to be linear. Also, note that in this research the satisfactory degree $\mu(f)=1$ if there exists $f^{\min }=f^{\max }$.

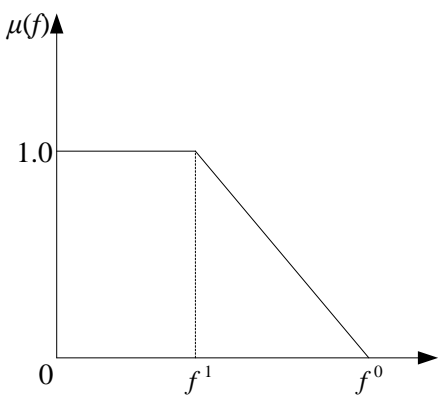

Fig. 2. Linear membership function

(1)The membership function of the leader

The individual best objective value of the leader is:

$$
f^{1}=f^{\min }=\min \left\{f^{(1)}\left(x, y_{1}, \ldots, y_{n}, z_{11}, \ldots, z_{1 m_{1}}, \ldots, z_{n 1}, \ldots, z_{n m_{n}}\right):\left(x, y_{1}, \ldots, y_{n}, z_{11}, \ldots, z_{1 m_{1}}, \ldots, z_{n 1}, \ldots, z_{n m_{n}}\right) \in S\right\} .
$$

The individual worst objective value is:

$$
f^{0}=f^{\max }=\max \left\{f^{(1)}\left(x, y_{1}, \ldots, y_{n}, z_{11}, \ldots, z_{1 m_{1}}, \ldots, z_{n 1}, \ldots, z_{n m_{n}}\right):\left(x, y_{1}, \ldots, y_{n}, z_{11}, \ldots, z_{1 m_{1}}, \ldots, z_{n 1}, \ldots, z_{n m_{n}}\right) \in S\right\} .
$$

The corresponding linear membership function $\mu\left(f^{(1)}\right)$ is defined as:

$$
\mu\left(f^{(1)}\right)=\left\{\begin{array}{cl}
0, & f^{(1)} \geq f^{0}, \\
\frac{f^{(1)}-f^{0}}{f^{1}-f^{0},} & f^{1}<f^{(1)}<f^{0}, \\
1, & f^{(1)} \leq f^{1} .
\end{array}\right.
$$

$\mu\left(f^{(1)}\right)$ can be used to denote the satisfactory degree of the leader towards an objective value $f^{(1)}$. $\mu\left(f^{0}\right)=0$ implies that the satisfactory degree of the leader is 0 when the objective value $f^{(1)}=f^{0}$, while the objective value $f^{(1)}=f^{1}$ such that $\mu\left(f^{1}\right)=1$ means that the satisfactory degree of the leader becomes 1 .

(2) The membership function of the middle-level follower $i \quad(i=1,2, \cdots, n)$

The middle-level follower $i$ makes its decision under the given decisions $\left(x^{*}, y_{1}^{*}, \ldots, y_{i-1}^{*}, y_{i+1}^{*}, \ldots, y_{n}^{*}\right)$ from the leader and other middle-level followers, thus, its individual best objective value is:

$$
f_{i}^{1}=f_{i}^{\min }=\min \left\{f_{i}^{(2)}\left(x^{*}, y_{1}^{*}, \ldots, y_{i-1}^{*}, y_{i}, y_{i+1}^{*}, \ldots, y_{n}^{*}, z_{i 1}, \ldots, z_{i m_{i}}\right):\left(y_{i}, z_{i 1}, \ldots, z_{i m_{i}}\right) \in S_{i}\left(x^{*}, y_{1}^{*}, \ldots, y_{i-1}^{*}, y_{i+1}^{*}, \ldots, y_{n}^{*}\right)\right\} \text {. }
$$

The individual worst objective value is:

$$
f_{i}^{0}=f_{i}^{\max }=\max \left\{f_{i}^{(2)}\left(x^{*}, y_{1}^{*}, \ldots, y_{i-1}^{*}, y_{i}, y_{i+1}^{*}, \ldots, y_{n}^{*}, z_{i 1}, \cdots, z_{i m_{i}}\right):\left(y_{i}, z_{i 1}, \ldots, z_{i m_{i}}\right) \in S_{i}\left(x^{*}, y_{1}^{*}, \ldots, y_{i-1}^{*}, y_{i+1}^{*}, \ldots, y_{n}^{*}\right)\right\} \text {. }
$$


The corresponding linear membership function $\mu_{i}\left(f_{i}^{(2)}\right)$ is defined as:

$$
\mu_{i}\left(f_{i}^{(2)}\right)=\left\{\begin{array}{cl}
0, & f_{i}^{(2)} \geq f_{i}^{0}, \\
\frac{f_{i}^{(2)}-f_{i}^{0}}{f_{i}^{1}-f_{i}^{0},} & f_{i}^{1}<f_{i}^{(2)}<f_{i}^{0}, \\
1, & f_{i}^{(2)} \leq f_{i}^{1} .
\end{array}\right.
$$

We can use $\mu_{i}\left(f_{i}^{(2)}\right)$ to denote the satisfactory degree of the middle-level follower $i$ towards an objective value $f_{i}^{(2)} \cdot \mu_{i}\left(f_{i}^{0}\right)=0$ implies that the satisfactory degree of the middle-level follower $i$ is 0 when the objective value $f_{i}^{(2)}=f_{i}^{0}$, whereas the objective value $f_{i}^{(2)}=f_{i}^{1}$ such that $\mu_{i}\left(f_{i}^{1}\right)=1$ means that the satisfactory degree becomes 1 .

(3) The membership function of the bottom-level follower ij $\left(j=1,2, \ldots, m_{i}, i=1,2, \ldots, n\right)$

The bottom-level follower $i j$ makes its decision under the given decision $\left(x^{*}, y_{i}^{*}, z_{i 1}^{*}, \ldots, z_{i(j-1)}^{*}, z_{i(j+1)}^{*}, \ldots, z_{i m_{i}}^{*}\right)$ from the leader, the middle-level follower $i$ and its own counterparts, thus, its individual best objective value is:

$$
f_{i j}^{1}=f_{i j}^{\min }=\min \left\{f_{i j}^{(3)}\left(x^{*}, y_{i}^{*}, z_{i 1}^{*}, \ldots, z_{i(j-1)}^{*}, z_{i j}, z_{i(j+1)}^{*}, \ldots, z_{i m_{i}}^{*}\right): z_{i j} \in S_{i j}\left(x^{*}, y_{i}^{*}, z_{i 1}^{*}, \ldots, z_{i(j-1)}^{*}, z_{i(j+1)}^{*}, \ldots, z_{i m_{i}}^{*}\right)\right\} .
$$

The individual worst objective value is:

$$
f_{i j}^{0}=f_{i j}^{\max }=\max \left\{f_{i j}^{(3)}\left(x^{*}, y_{i}^{*}, z_{i 1}^{*}, \ldots, z_{i(j-1)}^{*}, z_{i j}, z_{i(j+1)}^{*}, \ldots, z_{i m_{i}}^{*}\right): z_{i j} \in S_{i j}\left(x^{*}, y_{i}^{*}, z_{i 1}^{*}, \ldots, z_{i(j-1)}^{*}, z_{i(j+1)}^{*}, \ldots, z_{i m_{i}}^{*}\right)\right\} .
$$

The corresponding linear membership function $\mu_{i j}\left(f_{i j}^{(3)}\right)$ is defined as:

$$
\mu_{i j}\left(f_{i j}^{(3)}\right)=\left\{\begin{array}{cl}
0, & f_{i j}^{(3)} \geq f_{i j}^{0}, \\
\frac{f_{i j}^{(3)}-f_{i j}^{0}}{f_{i j}^{1}-f_{i j}^{0}}, & f_{i j}^{1}<f_{i j}^{(3)}<f_{i j}^{0}, \\
1, & f_{i j}^{(3)} \leq f_{i j}^{1} .
\end{array}\right.
$$

We can also use $\mu_{i j}\left(f_{i j}^{(3)}\right)$ to denote the satisfactory degree of the bottom-level follower $i j$ towards an objective value $f_{i j}^{(3)} \cdot \mu_{i j}\left(f_{i j}^{0}\right)=0$ implies that the satisfactory degree is 0 when the objective value $f_{i j}^{(3)}=f_{i j}^{0}$, while the objective value $f_{i j}^{(3)}=f_{i j}^{1}$ such that $\mu_{i j}\left(f_{i j}^{1}\right)=1$ means that the satisfactory degree is 1.

We use the proposed approach to evaluate the solutions enumerated in Example 1. For the leader, $f^{1}=f^{\min }=f^{(1)}(1,1,0.5,0.5,4.5,0,2)=3$ and $f^{0}=f^{\max }=f^{(1)}(0,1,0.5,3,3,0,2.5)=9.5$, thus, by the formula (14) the leader's satisfactory degrees are 1.0, 0.92 and 0.62 respectively towards the vertices $\left(x^{1}, y_{1}^{1}, y_{2}^{1}, z_{11}^{1}, z_{12}^{1}, z_{21}^{1}, z_{22}^{1}\right)=(1,1,0.5,0.5,4.5,0,2),\left(x^{2}, y_{1}^{2}, y_{2}^{2}, z_{11}^{2}, z_{12}^{2}, z_{21}^{2}, z_{22}^{2}\right)=(1,1,0.5,1,4,0,2.5)$ and $\left(x^{3}, y_{1}^{3}, y_{2}^{3}, z_{11}^{3}, z_{12}^{3}\right.$, $\left.z_{21}^{3}, z_{22}^{3}\right)=(1,1,0.5,2,3,1,1.5)$. By the formulas (15) and (16), the satisfactory degrees of multiple followers towards each solution are presented in Table 3. 
Table 3 Objective values and corresponding satisfactory degrees of decision entities in Example 1

\begin{tabular}{|c|c|c|c|c|c|c|c|c|c|c|c|c|c|c|}
\hline \multirow{2}{*}{ Vertex } & \multicolumn{2}{|c|}{ Leader } & \multicolumn{2}{|c|}{ Follower 1} & \multicolumn{2}{|c|}{ Follower 2} & \multicolumn{2}{|c|}{ Follower 11} & \multicolumn{2}{|c|}{ Follower 12} & \multicolumn{2}{|c|}{ Follower 21} & \multicolumn{2}{|c|}{ Follower 22} \\
\hline & $f^{(1)}$ & $\mu\left(f^{(1)}\right)$ & $f_{1}^{(2)}$ & $\mu_{1}\left(f_{1}^{(2)}\right)$ & $f_{2}^{(2)}$ & $\mu_{2}\left(f_{2}^{(2)}\right)$ & $f_{11}^{(3)}$ & $\mu_{11}\left(f_{11}^{(3)}\right)$ & $f_{12}^{(3)}$ & $\mu_{12}\left(f_{12}^{(3)}\right)$ & $f_{21}^{(3)}$ & $\mu_{21}\left(f_{21}^{(3)}\right)$ & $f_{22}^{(3)}$ & $\mu_{22}\left(f_{22}^{(3)}\right)$ \\
\hline$(1,1,0.5,0.5,4.5,0,2)$ & 3.0 & 1.0 & 5.5 & 1.0 & 3.0 & 1.0 & 7.5 & 0.83 & 8.0 & 1.0 & 3.5 & 0 & 0 & 0.80 \\
\hline$(1,1,0.5,1,4,0,2.5)$ & 3.5 & 0.92 & 5.5 & 1.0 & 3.5 & 0.67 & 8.0 & 0.67 & 8.0 & 1.0 & 4.0 & 0 & -0.5 & 1.0 \\
\hline$(1,1,0.5,2,3,1,1.5)$ & 5.5 & 0.62 & 5.5 & 1.0 & 3.5 & 0.67 & 9.0 & 1.0 & 8.0 & 1.0 & 0 & 1.0 & 2.5 & 1.0 \\
\hline
\end{tabular}

As we can see from Table 3, the vertex $\left(x^{1}, y_{1}^{1}, y_{2}^{1}, z_{11}^{1}, z_{12}^{1}, z_{21}^{1}, z_{22}^{1}\right)=(1,1,0.5,0.5,4.5,0,2)$ is the individual best solution to the leader such that the satisfactory degree is 1.0 , thus the leader anticipates that the followers can select $\left(y_{1}, y_{2}, z_{11}, z_{12}, z_{21}, z_{22}\right)=(1,0.5,0.5,4.5,0,2)$ to respond to its own decision $x=1$. Under the decision $x=1$ given by the leader, the middle-level followers make their decisions $\left(y_{1}, y_{2}\right)=(1,0.5)$ as desired by the leader, and they also desire that the bottom-level followers can react to the given decision $\left(x, y_{1}, y_{2}\right)=(1,1,0.5)$ by determining $\left(z_{11}, z_{12}, z_{21}, z_{22}\right)=(0.5,4.5,0,2)$ because their satisfactory degrees are both 1.0 under the solution. However, in view of the given decision by the leader and the middle-level follower 1, the bottom-level followers 11 and 12 will not choose the decision $\left(z_{11}, z_{12}\right)=(0.5,4.5)$ that are desired by the leader and the middle-level follower 1 since they still have space to optimize their objectives and improve their satisfactory degrees. Thus, $\left(z_{11}, z_{12}\right)=(0.5,4.5)$ is not an optimal solution to the bottom-level followers 11 and 12 and they will select $\left(z_{11}, z_{12}\right)=(2,3)$ to achieve the highest satisfactory degree 1.0, which also is the Nash equilibrium between them under the decision made by the leader and the middle-level follower 1. Similarly, the bottom-level followers 21 and 22 will make the decision $\left(z_{21}, z_{22}\right)=(1,1.5)$ to respond to the leader and the middle-level follower 2 . The leader and the middle-level followers have to reduce their individual satisfactory degrees to bend to the increase in the satisfactory degrees of the bottom-level followers throughout the MFTL decision-making process. In this way, the decision entities finally achieve a an optimal solution $\left(x^{3}, y_{1}^{3}, y_{2}^{3}, z_{11}^{3}, z_{12}^{3}, z_{21}^{3}, z_{22}^{3}\right)=(1,1,0.5,2,3,1,1.5)$, under which the satisfactory degrees of all the bottom-level followers go up to 1.0.

Although the satisfactory degrees of the leader and the middle-level follower 2 drop to 0.62 and 0.67 respectively, the numbers become the highest satisfactory degrees for them under the Stackelberg-Nash equilibrium and the uncooperative relationship among all decision entities. In real-world cases, the situation indicates that higher satisfactory degrees of the leader and the middle-level follower 2 cannot be achieved under the current decision conditions unless they may persuade the bottom-level followers to cooperate with them and to reduce the corresponding satisfactory degrees. For example, if the bottom-level followers 11, 21 and 22 are willing to accept their respective satisfactory degrees $0.83,0$ and 0.80 , the solution to Example 1 would be $\left(x^{1}, y_{1}^{1}, y_{2}^{1}, z_{11}^{1}, z_{12}^{1}, z_{21}^{1}, z_{22}^{1}\right)=(1,1,0.5,0.5,4.5,0,2)$, which ensures that the corresponding satisfactory degrees the leader and the bottom-level follower 2 rise up to 1.0. Otherwise, they have to adjust the current decision context through changing objective functions or constraint conditions to generate a new round of the 
decision-making process. We will illustrate how to adopt the evaluation criterion to deal with real-world MFTL decision problems through a case study in the following Section 5.

\section{A case study: Production-inventory planning}

In this section, we will use a case study on production-inventory planning to illustrate the proposed MFTL decision techniques.

\subsection{Case description}

Nowadays, manufacturers usually work in a distributed or decentralized manner in a complex supply chain network comprising of suppliers, sales and logistics companies, customers and other specialized service functions [7]. Researchers as well as practitioners in manufacturing industries have placed importance on developing production and inventory control capabilities to enhance their market position in supply chain management [22], which demands that manufacturing enterprises have to make right decisions on scheduling of their production and allocation of inventory to satisfy market requirements, shorten delivery time and reduce total production costs $[2,8,28]$. Therefore, it is increasingly important to have efficient and easily-applicable models and solution methods to describe and solve related production-inventory decision problems $[6,13]$ although modeling deception in a real-world conflict situation is usually difficult [16].

In this section, we adopt the proposed MFTL decision techniques to handle a production-inventory planning problem within a real-world conglomerate enterprise. The conglomerate is composed of a sales company, two logistics centers and two manufacturing factories attached to each logistics center, which are distributed throughout a three-stage hierarchical supply chain. The three-level hierarchical structure of the conglomerate is shown in Fig. 3. Specifically, the sales company covers products marketing of the enterprise and has an independent products warehouse to satisfy market demand and shorten time-to-market. Both logistics centers also hold a certain amount of products inventory to respond to market requirements and reduce the inventory pressure of the sales company. According to market requirements and the holding inventories of the sales company and the logistics centers, the manufacturing factories are responsible for the production organization involving making detailed production plans and executing production activities.

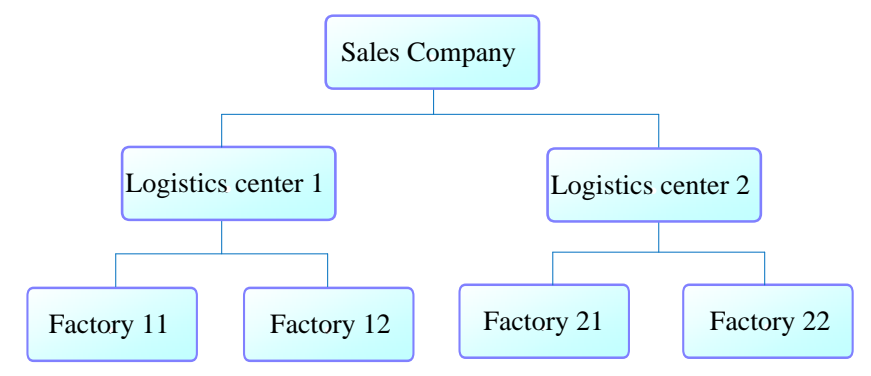

Fig. 3. Hierarchical structure of the conglomerate enterprise

The decision situation we study in this paper is described as follows. During a peak season of products sales, the market requirements exceed the normal supply capacity of the enterprise so that four manufacturing factories have to organize overtime production. The sum of overtime outputs produced by four factories and safety stocks held by the sales company and two logistics centers are demanded to satisfy 
the exceeded market requirements. The more safety stocks imply the fewer overtime production outputs, but also mean the more inventory holding costs. Under the given market requirements, the decision entities distributed throughout the three-level hierarchy try to minimize their individual costs by considering their own constraints and implicit decisions made by other decision entities. More specifically, the sales company at the top level has the priority to determine its safety stock to minimize its inventory holding cost by considering the given market requirements and implicit reactions of other decision entities. In view of the decision made by the sales company, the logistics centers at the middle level then determine their individual safety stocks to minimize their own inventory holding costs by considering their own constraints and implicit reactions of their subordinate factories. Finally, each manufacturing factory at the bottom level makes overtime production plans in the light of the inventories held by the top and middle levels.

Furthermore, to reduce the total cost of the conglomerate, the conglomerate anticipates that decision entities whose inventory holding cost or overtime production cost is lower are able to keep more inventories or manufacture more production outputs. Thus, the conglomerate makes some management strategies to intervene and reconcile the decision process of its subordinate decision entities. For example, the conglomerate claims that each logistics center should take the inventory determined by the other logistics center as a reference when making its own decisions. If the inventory of a logistics center is less than the other, it means the less inventory holding cost but implies that the logistics center is demanded to undertake an opportunity cost for its own decision on holding less inventory. Also, each factory needs to reference the production plans made by other counterparts attached to the same logistics center when making its own production plans. If the production outputs of a factory are less than the other, it means the less overtime production costs but implies that the factory needs to cover an opportunity cost for its own decision on less production outputs. In addition, the sales company is demanded to afford the marketing cost and backlogging cost of the conglomerate. However, to reduce the total cost to the sales company, the conglomerate demands that both logistics centers must share part of the inventory holding cost and compensate for the marketing cost of the sales company. Similarly, to reduce the pressure of overtime production, the factories at the bottom level are also demanded to compensate the inventory holding cost of their superior logistics center to encourage it to keep more safety stocks. Therefore, under the current decision situation, the decision entities will try to minimize their individual overall costs by making their individual decisions, and the decision processes are executed sequentially, interactively and repeatedly within the tri-level hierarchy until the Stackelberg-Nash equilibrium is achieved among them.

This case clearly describes a MFTL decision process which includes one leader (the sales company), two middle-level followers (the logistics centers) and two bottom-level followers (the manufacturing factories) attached to each middle-level follower. The leader, the middle-level followers and the bottom-level followers make their individual decisions in sequence, and each decision entity cannot control decisions of the others but is affected by their reactions. It is noticeable that the multiple middle-level and bottom-level followers also consider decisions made by their counterparts as references, which implies a reference-uncooperative relationship among the multiple followers at both the middle and bottom levels. The case can thus be considered as a reference-uncooperative MFTL decision problem. 
In the light of the above problem description, let $n=2$ be the number of logistics centers, and $i$ be the index for logistics centers, $i=1,2$; while let $m_{i}=2$ be the number of manufacturing factories attached to the logistics center $i$, and $j$ be the index for manufacturing factories, $j=1,2$. To model the problem conveniently, related notations of decision variables and some key parameters in the scenario are shown in Table 4.

Table 4 Symbols for decision variables and parameters employed

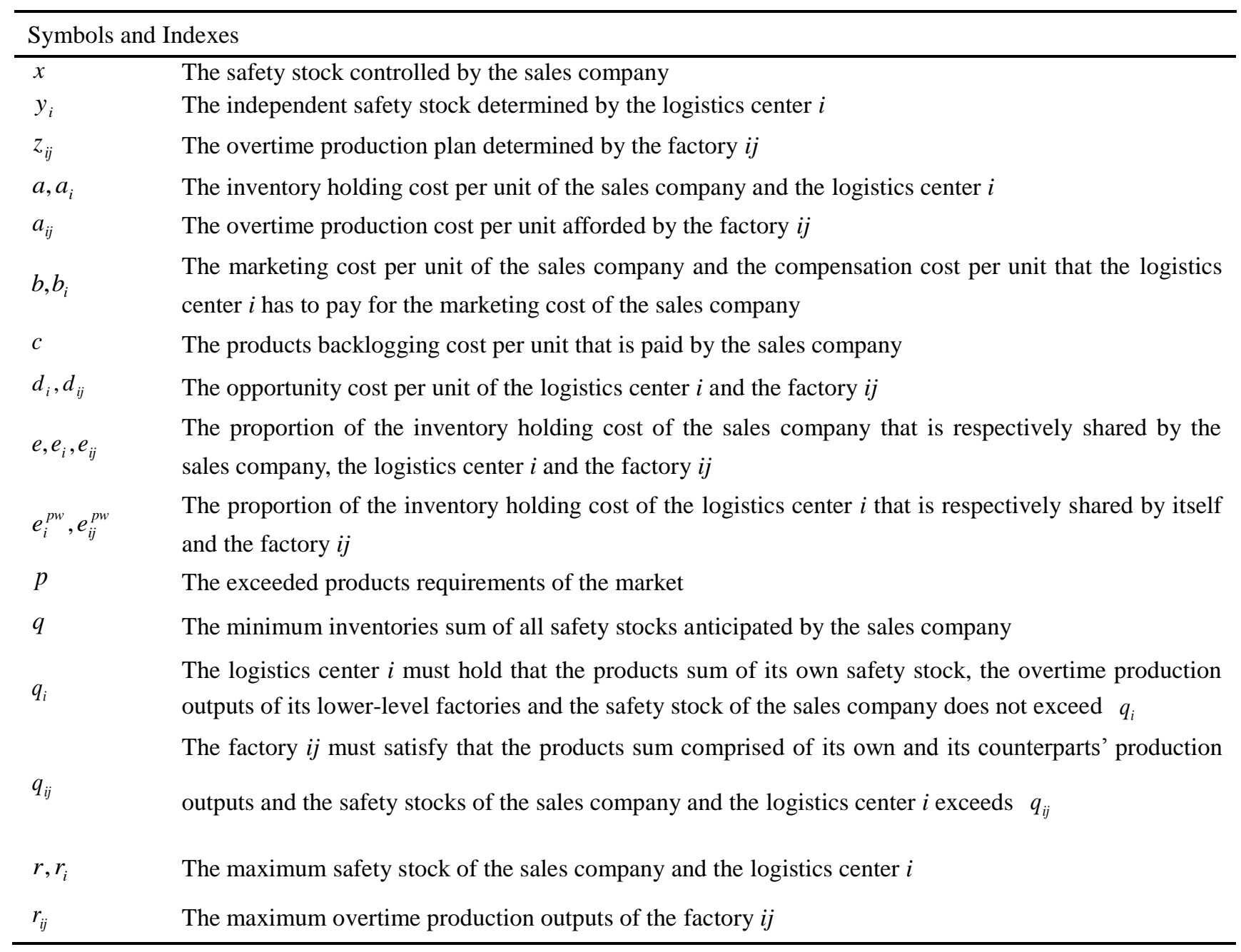

\subsection{Model building}

Based on the above decision conditions and strategies, the MFTL decision model of the case is established as follows in the form of the general model (1) proposed in Section 2.1.

(1) The decision problem of the sales company (top-level leader)

$$
\begin{aligned}
\min _{x \in X} f^{(1)}\left(x, y_{1}, y_{2}, z_{11}, z_{12}, z_{21}, z_{22}\right)=a e x+b p+c\left(x+\sum_{i=1}^{2} y_{i}+\sum_{i=1}^{2} \sum_{j=1}^{2} z_{i j}-p\right)-\sum_{i=1}^{2} b_{i}\left(y_{i}+\sum_{j=1}^{2} z_{i j}\right) \\
\text { s.t. } x+\sum_{i=1}^{2} y_{i}+\sum_{i=1}^{2} \sum_{j=1}^{2} z_{i j} \geq p \\
\quad x+\sum_{i=1}^{2} y_{i} \geq q \\
0 \leq x \leq r .
\end{aligned}
$$


The sales company's objective function (17a) involves its safety stock's inventory holding cost aex, the marketing cost $b p$, the backlogging cost $c\left(x+\sum_{i=1}^{2} y_{i}+\sum_{i=1}^{2} \sum_{j=1}^{2} z_{i j}-p\right)$ and the minus marketing compensation cost $\sum_{i=1}^{2} b_{i}\left(y_{i}+\sum_{j=1}^{2} z_{i j}\right)$ derived from the logistics centers. Constraint condition (17b) means the upper bound of the products sum of all safety stocks and overtime production outputs of all manufacturing factories, while constraint condition (17c) implies the upper bound of the sum of all safety stocks. Constraint condition (17d) represents the lower and upper limits to the sales company's safety stock.

(2) The decision problem of the logistics center (middle-level follower) $i$ for $i=1,2$

$$
\begin{aligned}
& \min _{y_{i} \in Y_{i}} f_{i}^{(2)}\left(x, y_{1}, y_{2}, z_{i 1}, z_{i 2}\right)=a_{i} e_{i}^{p w} y_{i}+d_{i} \Delta y_{i}+a e_{i} x+b_{i}\left(y_{i}+\sum_{j=1}^{2} z_{i j}\right) \\
& \text { s.t. } \quad x+\sum_{i=1}^{2} y_{i}+\sum_{j=1}^{2} z_{i j} \leq q_{i}, \\
& \quad 0 \leq y_{i} \leq r_{i} .
\end{aligned}
$$

The objective function (17e) of the logistics center $i$ involves its safety stock holding cost $a_{i} e_{i}^{p w} y_{i}$, the opportunity cost $d_{i} \Delta y_{i}$, the shared inventory holding cost $a e_{i} x$ of the sales company's safety stock and the marketing compensation cost $b_{i}\left(y_{i}+\sum_{j=1}^{2} z_{i j}\right)$ paid to the sales company. Note that $\Delta y_{1}=y_{2}-y_{1}$ and $\Delta y_{2}=y_{1}-y_{2}$. Constraint condition (17f) reflects the upper bound of the products sum consisting of all safety stocks and the overtime production outputs of the manufacturing factories attached to the middle-level follower $i$. Constraint condition $(17 \mathrm{~g})$ represents the lower and upper limits to the safety stock of the logistics center $i$.

(3) The decision problem of the manufacturing factory (bottom-level follower) $i j$ for $j=1,2, i=1,2$

$$
\begin{aligned}
& \min _{z_{i j} \in Z_{i j}} f_{i j}^{(3)}\left(x, y_{i}, z_{i 1}, z_{i 2}\right)=a_{i j} z_{i j}+d_{i j} \Delta z_{i j}+a e_{i j} x+a_{i} e_{i j}^{p w} y_{i} \\
& \text { s.t. } \quad x+y_{i}+\sum_{j=1}^{2} z_{i j} \geq q_{i j} \\
& \quad 0 \leq z_{i j} \leq r_{i j} .
\end{aligned}
$$

The objective function (17h) of the manufacturing factory $i j$ involves its overtime production cost $a_{i j} z_{i j}$, the opportunity cost $d_{i j} \Delta z_{i j}$, and the shared inventory holding cost $a e_{i j} x$ and $a_{i} e_{i j}^{p w} y_{i}$ respectively for the safety stocks of the sales company and the logistics center $i$. Note that $\Delta z_{i 1}=z_{i 2}-z_{i 1}$ and $\Delta z_{i 2}=z_{i 1}-z_{i 2}$. Constraint condition (17i) reflects the upper bound of the products sum consisting of overtime production outputs of the manufacturing factories attached to the logistics center $i$ and safety stocks of the sales company and the logistics center $i$. Constraint condition (17j) represents the lower and upper limits to the overtime production outputs of the manufacturing factory $i j$. 
This MFTL decision model (17) describes the real-world production and inventory decision problem which is a concretization of the general model (1) proposed in Section 2.1. We then adopt the MFTL $K$ th-Best algorithm to solve the model by a numerical experiment.

\subsection{Numerical experiment and results analysis}

This section shows the computational results achieved by the proposed MFTL Kth-Best algorithm and evaluation of solutions. The experimental data employed for the model (17) is provided in Tables 5-7.

Table 5 Data for the sales company

\begin{tabular}{ccccccc}
\hline$a$ & $b$ & $c$ & $e$ & $p$ & $q$ & $r$ \\
\hline 5.0 & 2.0 & 2.0 & 0.20 & 9.0 & 3.5 & 2.5 \\
\hline
\end{tabular}

Table 6 Data for the logistics centers

\begin{tabular}{cccccccc}
\hline$i$ & $a_{i}$ & $b_{i}$ & $d_{i}$ & $e_{i}$ & $e_{i}^{p w}$ & $q_{i}$ & $r_{i}$ \\
\hline 1 & 4.0 & 1.0 & 4.0 & 0.20 & 0.50 & 8.0 & 1.0 \\
2 & 4.0 & 3.0 & 4.0 & 0.20 & 0.50 & 6.0 & 0.50 \\
\hline
\end{tabular}

Table 7 Data for the manufacturing factories

\begin{tabular}{llllllll}
\hline$i$ & $j$ & $a_{i j}$ & $d_{i j}$ & $e_{i j}$ & $e_{i j}^{p w}$ & $q_{i j}$ & $r_{i j}$ \\
\hline 1 & 1 & 1.0 & 2.0 & 0.10 & 0.25 & 7.0 & 3.0 \\
1 & 2 & 3.0 & 2.0 & 0.10 & 0.25 & 7.0 & 3.0 \\
2 & 1 & 2.0 & 3.0 & 0.10 & 0.25 & 4.0 & 1.0 \\
2 & 2 & 4.0 & 3.0 & 0.10 & 0.25 & 4.0 & 2.0 \\
\hline
\end{tabular}

We use a tri-level decision support system to build the mathematics formula of model (17) based on the experimental data in Tables 5-7, shown as Fig. 4.

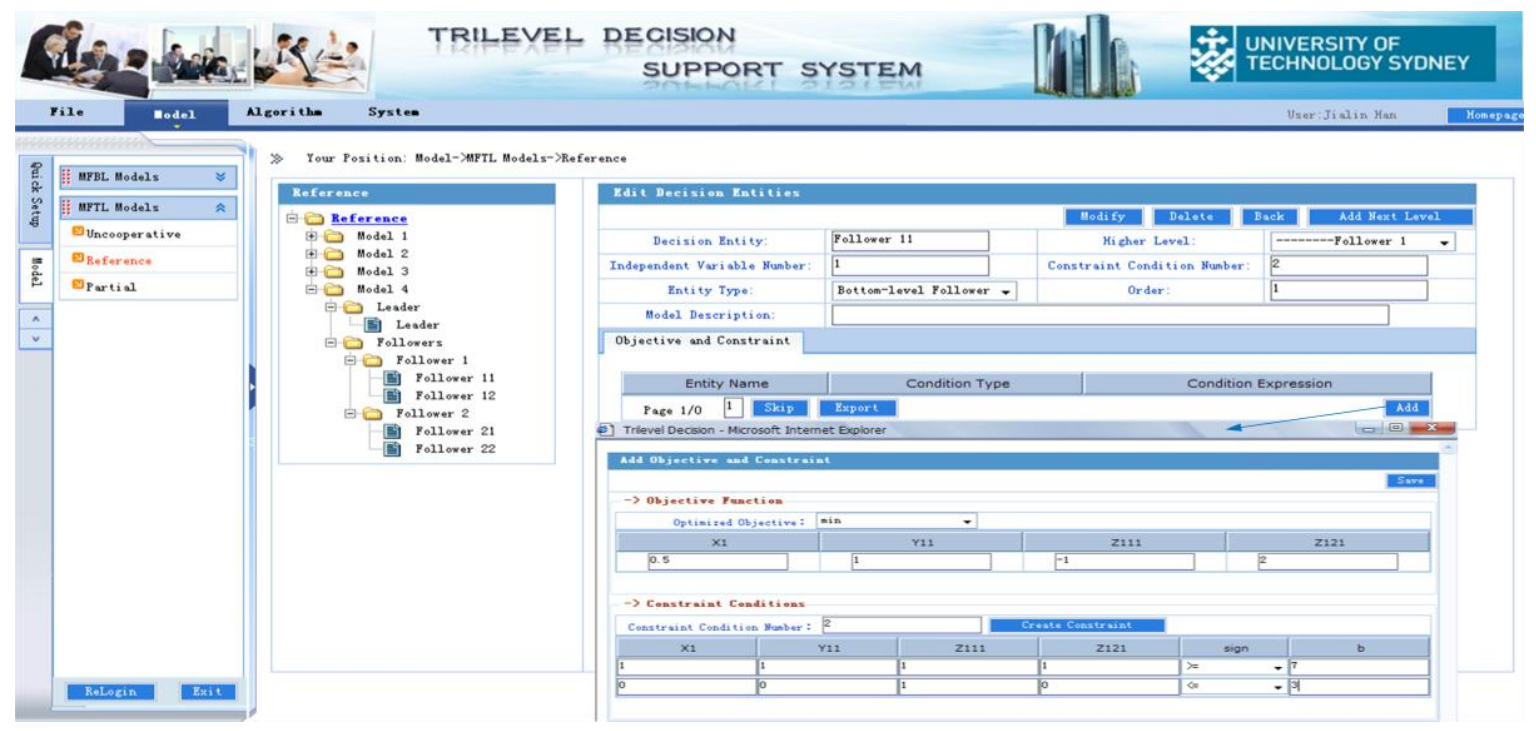

Fig. 4. Model building of the MFTL decision problem

The tri-level decision support system driven by the MFTL Kth-Best algorithm then finds an optimal solution (also called a Stackelberg-Nash solution) to the model (17) shown in Fig. 5, which clearly shows that a solution is attained at the vertex $\left(x, y_{1}, y_{2}, z_{11}, z_{12}, z_{21}, z_{22}\right)=(2,1,0.5,3,1,1,0.5)$ and the objective 
values of all decision entities are $f^{(1)}=9.0, f_{1}^{(2)}=7.0, f_{2}^{(2)}=11.0, f_{11}^{(3)}=1.0, f_{12}^{(3)}=9.0, f_{21}^{(3)}=2.0, f_{22}^{(3)}=5.0$. Fig. 5 also displays that an optimal solution is found after 12 iterations and the computing process has spent 862 milliseconds of CPU time. 12 iterations imply that we must search 12 vertices at least to find an optimal solution, and this task would take a long time if the computing was conducted manually as well as solving the Example 1 in Section 3. The convenience of a decision support system is its very short computing time especially for solving complex MFTL decision problems in applications. Also, the decision support systems can output the detailed computing process driven by the MFTL Kth-Best algorithm, which can help us to analysis the satisfactory degree of decision entities towards solutions and can provide references for decision conditions adjustment in the real-world case. In addition, the web-based decision support system has another advantage that it can be integrated with other application systems commonly implemented in manufacturing enterprises, business organizations and governments, such as ERP, MES, e-business systems and common service platforms, which provides an opportunity to share data with other systems and support the decision-making of other systems. In this way, the tri-level decision support system is able to be a convenient means of decision-making in applications.

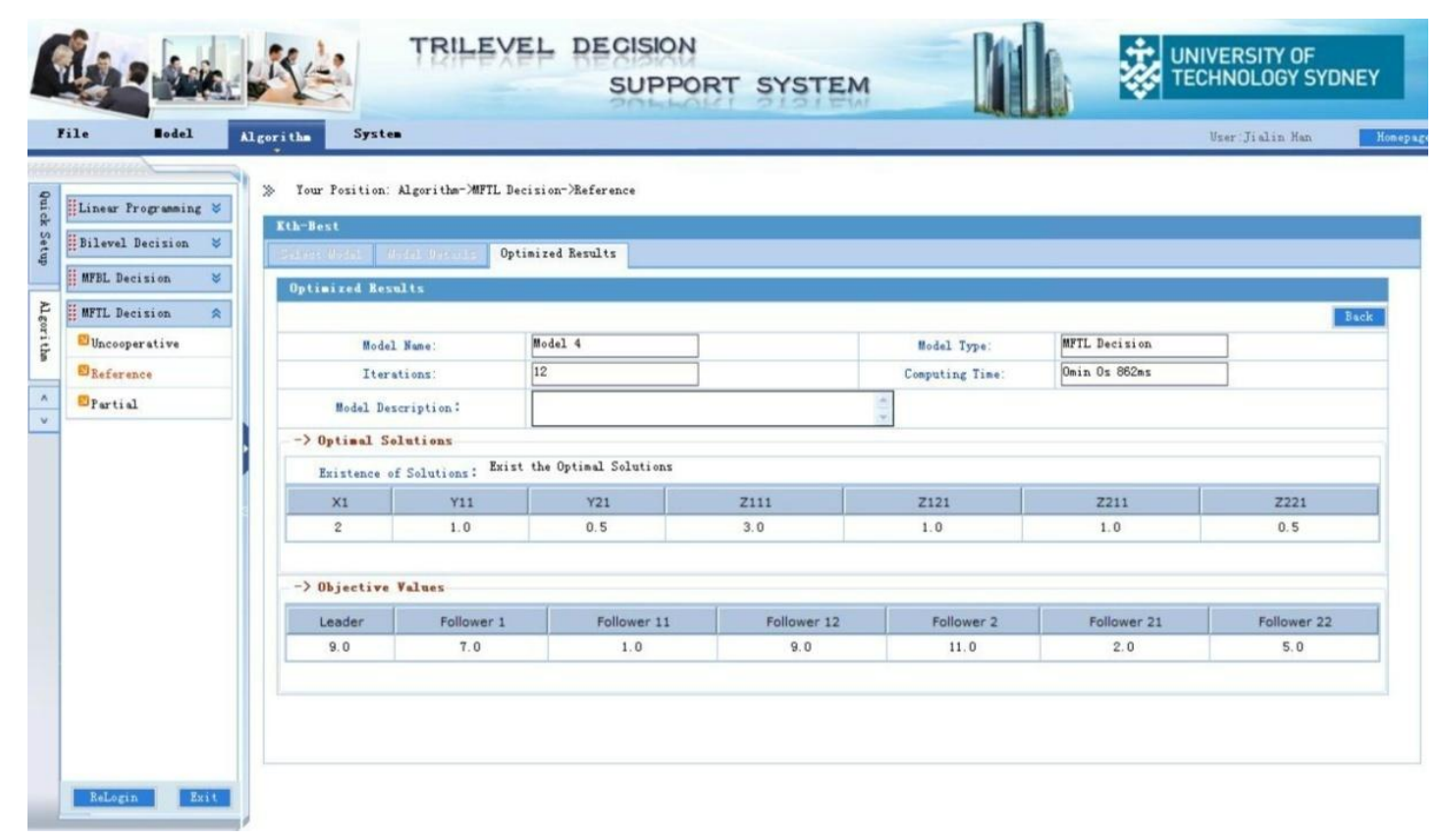

Fig. 5. Computing results display of the MFTL decision problem

The detailed computing process driven by the MFTL Kth-Best algorithm is shown in Table 8 which includes related data and parameters generated in the computing process. Specifically, Table 8 presents the vertex $s^{k}$ that is searched in the current iteration $k$, and the adjacent vertices set $W_{k}$ of the current vertex $s^{k}$. $T$ represents the set of vertices that have been searched in the past iterations while $W$ is the set of vertices that are needed to verify whether or not an optimal solution occurs inside in the following iteration. Following procedures of the MFTL Kth-Best algorithm, we finally obtain an optimal solution after 12 iterations. Note that $W_{7}=\varnothing, W_{8}=\varnothing$ and $W_{10}=\varnothing$ in Table 8 do not mean that adjacent vertices of $s^{7}, s^{8}$ and $s^{10}$ do not exist, but imply that their adjacent vertices have been found in previous iterations and have been involved in 
$W$.

Table 8 The detailed computing process of the MFTL $K$ th-Best algorithm

\begin{tabular}{|c|c|c|c|c|}
\hline Iteration $k$ & $s^{k}=\left(x^{k}, y_{1}^{k}, y_{2}^{k}, z_{11}^{k}, z_{12}^{k}, z_{21}^{k}, z_{22}^{k}\right)$ & $W_{k}$ & $T$ & $W$ \\
\hline 1 & $(2,1,0.5,1,3,0.5,2)$ & $\begin{array}{l}\{(2.5,1,0,0.5,3,0.5,2) \\
(2,1,0.5,1,3,0,2) \\
(2.5,1,0.5,0.5,3,0,2) \\
(2,1,0.5,3,1,0.5,2) \\
(2,1,0.5,1,3,1,1.5) \\
(2.5,0.5,0.5,1,3,0.5,2)\}\end{array}$ & $\left\{s^{1}\right\}$ & $W_{1}$ \\
\hline 2 & $(2,1,0.5,3,1,0.5,2)$ & $\begin{array}{l}\{(2.5,1,0,3,0.5,0.5,2) \\
(2,1,0.5,3,1,0,2) \\
(2.5,1,0.5,3,0.5,0,2) \\
(2,1,0.5,3,1,1,1.5) \\
(2.5,0.5,0.5,3,1,0.5,2)\}\end{array}$ & $\left\{s^{1}, s^{2}\right\}$ & $\left(W \cup W_{2}\right) \backslash T$ \\
\hline 3 & $(2,1,0.5,1,3,1,1.5)$ & $\begin{array}{l}\{(2.5,1,0,0.5,3,1,1.5) \\
(2.5,0.5,0.5,1,3,1,1.5) \\
(2.5,1,0.5,0.5,3,1,1) \\
(2,1,0.5,1,3,1,0.5)\}\end{array}$ & $\left\{s^{1}, s^{2}, s^{3}\right\}$ & $\left(W \cup W_{3}\right) \backslash T$ \\
\hline 4 & $(2,1,0.5,3,1,1,1.5)$ & $\begin{array}{l}\{(2.5,1,0,3,0.5,1,1.5) \\
(2.5,0.5,0.5,3,1,1,1.5) \\
(2.5,1,0.5,3,0.5,1,1) \\
(2,1,0.5,3,1,1,0.5)\}\end{array}$ & $\left\{s^{1}, s^{2}, s^{3}, s^{4}\right\}$ & $\left(W \cup W_{4}\right) \backslash T$ \\
\hline 5 & $(2,1,0.5,1,3,0,2)$ & $\begin{array}{c}\{(2.5,1,0,0.5,3,0,2) \\
(2.5,0.5,0.5,1,3,0,2) \\
(2,1,0.5,1,3,0,1.5)\}\end{array}$ & $\left\{s^{1}, s^{2}, s^{3}, s^{4}, s^{5}\right\}$ & $\left(W \cup W_{5}\right) \backslash T$ \\
\hline 6 & $(2,1,0.5,3,1,0,2)$ & $\begin{array}{c}\{(2.5,1,0,3,0.5,0,2) \\
(2.5,0.5,0.5,3,1,0,2) \\
(2,1,0.5,3,1,0,1.5)\}\end{array}$ & $\left\{s^{1}, s^{2}, s^{3}, s^{4}, s^{5}, s^{6}\right\}$ & $\left(W \cup W_{6}\right) \backslash T$ \\
\hline 7 & $(2.5,0.5,0.5,1,3,0.5,2)$ & $\varnothing$ & $\left\{s^{1}, s^{2}, s^{3}, s^{4}, s^{5}, s^{6}, s^{7}\right\}$ & $\left(W \cup W_{7}\right) \backslash T$ \\
\hline 8 & $(2.5,0.5,0.5,3,1,0.5,2)$ & $\varnothing$ & $\left\{s^{1}, s^{2}, s^{3}, s^{4}, s^{5}, s^{6}, s^{8}\right\}$ & $\left(W \cup W_{8}\right) \backslash T$ \\
\hline 9 & $(2.5,0.5,0.5,1,3,1,1.5)$ & $\{(2.5,0.5,0.5,1,3,1,0.5)\}$ & $\left\{s^{1}, s^{2}, s^{3}, s^{4}, s^{5}, s^{6}, s^{8}, s^{9}\right\}$ & $\left(W \cup W_{9}\right) \backslash T$ \\
\hline 10 & $(2,1,0.5,1,3,1,0.5)$ & $\varnothing$ & $\left\{s^{1}, s^{2}, s^{3}, s^{4}, s^{5}, s^{6}, s^{8}, s^{9}, s^{10}\right\}$ & $\left(W \cup W_{10}\right) \backslash T$ \\
\hline 11 & $(2.5,0.5,0.5,3,1,1,1.5)$ & $\{(2.5,0.5,0.5,3,1,1,0.5)\}$ & $\left\{s^{1}, s^{2}, s^{3}, s^{4}, s^{5}, s^{6}, s^{8}, s^{9}, s^{10}, s^{11}\right\}$ & $\left(W \cup W_{11}\right) \backslash T$ \\
\hline 12 & $(2,1,0.5,3,1,1,0.5)$ & --- & -- & --- \\
\hline
\end{tabular}

Table 9 displays the objective values of all decision entities respectively towards each solution enumerated by the MFTL Kth-Best algorithm, while Table 10 shows the corresponding satisfactory degrees that are computed by the formulas (14), (15) and (16). As we can see from Table 9, under the current decision context within the conglomerate enterprise, $\left(x^{1}, y_{1}^{1}, y_{2}^{1}, z_{11}^{1}, z_{12}^{1}, z_{21}^{1}, z_{22}^{1}\right)=(2,1,0.5,1,3,0.5,2)$, $\left(x^{2}, y_{1}^{2}, y_{2}^{2}, z_{11}^{2}, z_{12}^{2}, z_{21}^{2}, z_{22}^{2}\right)=(2,1,0.5,3,1,0.5,2),\left(x^{3}, y_{1}^{3}, y_{2}^{3}, z_{11}^{3}, z_{12}^{3}, z_{21}^{3}, z_{22}^{3}\right)=(2,1,0.5,1,3,1,1.5)$ and $\left(x^{4}, y_{1}^{4}, y_{2}^{4}\right.$, $\left.z_{11}^{4}, z_{12}^{4}, z_{21}^{4}, z_{22}^{4}\right)=(2,1,0.5,3,1,1,1.5)$ are the individual best solutions to the leader (the sales company), which implies that the leader anticipates that the middle-level and bottom-level followers (the logistics centers and 
manufacturing factories) can choose $\left(y_{1}^{1}, y_{2}^{1}, z_{11}^{1}, z_{12}^{1}, z_{21}^{1}, z_{22}^{1}\right)=(1,0.5,1,3,0.5,2),\left(y_{1}^{2}, y_{2}^{2}, z_{11}^{2}, z_{12}^{2}, z_{21}^{2}, z_{22}^{2}\right)=$ $(1,0.5,3,1,0.5,2), \quad\left(y_{1}^{3}, y_{2}^{3}, z_{11}^{3}, z_{12}^{3}, z_{21}^{3}, z_{22}^{3}\right)=(1,0.5,1,3,1,1.5)$ or $\quad\left(y_{1}^{4}, y_{2}^{4}, z_{11}^{4}, z_{12}^{4}, z_{21}^{4}, z_{22}^{4}\right)=(1,0.5,3,1,1,1.5) \quad$ to respond to itself after it determined $x=2$. However, it can be seen from Table 10 that the middle-level follower 2 and the bottom-level followers 11,21, and 22 cannot always achieve individual best satisfactory degrees if they make the decisions desired by the leader. In the reference-uncooperative decision situation, the followers will choose $\left(y_{1}^{12}, y_{2}^{12}, z_{11}^{12}, z_{12}^{12}, z_{21}^{12}, z_{22}^{12}\right)=(1,0.5,3,1,1,0.5)$ to react to the leader's decision $x=2$ such that their satisfactory degrees all grow up to 1.0 .

Table 9 Solutions and objective values of decision entities

\begin{tabular}{|c|c|c|c|c|c|c|c|c|}
\hline Iteration $k$ & Vertex $s^{k}$ & $F$ & $f_{1}^{(2)}$ & $f_{2}^{(2)}$ & $f_{11}^{(3)}$ & $f_{12}^{(3)}$ & $f_{21}^{(3)}$ & $f_{22}^{(3)}$ \\
\hline 1 & $(2,1,0.5,1,3,0.5,2)$ & 8.0 & 7.0 & 14.0 & 7.0 & 7.0 & 7.0 & 5.0 \\
\hline 2 & $(2,1,0.5,3,1,0.5,2)$ & 8.0 & 7.0 & 14.0 & 1.0 & 9.0 & 7.0 & 5.0 \\
\hline 3 & $(2,1,0.5,1,3,1,1.5)$ & 8.0 & 7.0 & 14.0 & 7.0 & 7.0 & 5.0 & 6.0 \\
\hline 4 & $(2,1,0.5,3,1,1,1.5)$ & 8.0 & 7.0 & 14.0 & 1.0 & 9.0 & 5.0 & 6.0 \\
\hline 5 & $(2,1,0.5,1,3,0,2)$ & 8.5 & 7.0 & 12.5 & 7.0 & 7.0 & 7.5 & 3.5 \\
\hline 6 & $(2,1,0.5,3,1,0,2)$ & 8.5 & 7.0 & 12.5 & 1.0 & 9.0 & 7.5 & 3.5 \\
\hline 7 & $(2.5,0.5,0.5,1,3,0.5,2)$ & 9.0 & 8.0 & 12.5 & 6.75 & 6.75 & 7.25 & 5.25 \\
\hline 8 & $(2.5,0.5,0.5,3,1,0.5,2)$ & 9.0 & 8.0 & 12.5 & 0.75 & 8.75 & 7.25 & 5.25 \\
\hline 9 & $(2.5,0.5,0.5,1,3,1,1.5)$ & 9.0 & 8.0 & 12.5 & 6.75 & 6.75 & 5.25 & 6.25 \\
\hline 10 & $(2,1,0.5,1,3,1,0.5)$ & 9.0 & 7.0 & 11.0 & 7.0 & 7.0 & 2.0 & 5.0 \\
\hline 11 & $(2.5,0.5,0.5,3,1,1,1.5)$ & 9.0 & 8.0 & 12.5 & 0.75 & 8.75 & 5.25 & 6.25 \\
\hline 12 & $(2,1,0.5,3,1,1,0.5)$ & 9.0 & 7.0 & 11.0 & 1.0 & 9.0 & 2.0 & 5.0 \\
\hline
\end{tabular}

More specifically, for the given decision $\left(x, y_{1}\right)=(2,1)$ by the leader and the middle-level follower 1 , the bottom-level followers 11 and 12 achieve a Nash equilibrium solution $\left(z_{11}, z_{12}\right)=(3,1)$ to respond to the leader and the middle-level follower 1. Similarly, for the given decision $\left(x, y_{2}\right)=(2,0.5)$ by the leader and the middle-level follower 2, the bottom-level followers 21 and 22 achieve a Nash equilibrium solution $\left(z_{21}, z_{22}\right)=(1,0.5)$ to respond to the leader and the middle-level follower 2 . Therefore, $\left(y_{1}, z_{11}, z_{12}\right)=(1,3,1)$ and $\left(y_{2}, z_{21}, z_{22}\right)=(0.5,1,0.5)$ are Stackelberg-Nash solutions respectively for the middle-level follower $i$ $(i=1,2)$ and its bottom-level followers under the given decision $x=1$ by the leader. Also, for the given decision $x=2$ by the leader, $\left(y_{1}, y_{2}\right)=(1,0.5)$ is a Nash equilibrium solution for the middle-level followers while taking into account implicit reactions of their respective bottom-level followers. Therefore, $\left(x^{12}, y_{1}^{12}, y_{2}^{12}, z_{11}^{12}, z_{12}^{12}, z_{21}^{12}, z_{22}^{12}\right)=(2,1,0.5,3,1,1,0.5)$ is a Stackelberg-Nash solution to the production-inventory 
planning problem.

Table 10 The satisfactory degree of decision entities towards solutions

\begin{tabular}{|c|c|c|c|c|c|c|c|c|c|c|c|c|c|c|c|c|c|c|c|c|c|}
\hline \multirow{2}{*}{$\begin{array}{c}\text { Iteration } \\
k\end{array}$} & \multicolumn{3}{|c|}{ Leader } & \multicolumn{3}{|c|}{ Follower 1} & \multicolumn{3}{|c|}{ Follower 2} & \multicolumn{3}{|c|}{ Follower 11} & \multicolumn{3}{|c|}{ Follower 12} & \multicolumn{3}{|c|}{ Follower 21} & \multicolumn{3}{|c|}{ Follower 22} \\
\hline & $f^{1}$ & $f^{0}$ & $\mu\left(f^{(1)}\right)$ & $f_{1}^{1}$ & $f_{1}^{0}$ & $\mu_{1}\left(f_{1}^{(2)}\right)$ & $f_{2}^{1}$ & $f_{2}^{0}$ & $\mu_{2}\left(f_{2}^{(2)}\right)$ & $f_{11}^{1}$ & $f_{11}^{0}$ & $\mu_{11}\left(f_{11}^{(3)}\right)$ & $f_{12}^{1}$ & $f_{12}^{0}$ & $\mu_{12}\left(f_{12}^{(3)}\right)$ & $f_{21}^{1}$ & $f_{21}^{0}$ & $\mu_{21}\left(f_{21}^{(3)}\right)$ & $f_{22}^{1}$ & $f_{22}^{0}$ & $\mu_{22}\left(f_{22}^{(3)}\right)$ \\
\hline 1 & 8.0 & 11.5 & 1.0 & 7.09 & 9.5 & 1.0 & 11.0 & 15.0 & 0.25 & 5.0 & 7.0 & 0 & 7.0 & 7.0 & 1.0 & 6.5 & 7.5 & 0.5 & 4.0 & 5.0 & 0 \\
\hline 2 & 8.0 & 11.5 & 1.0 & 7.09 & 9.5 & 1.0 & 11.0 & 15.0 & 0.25 & 1.0 & 1.0 & 1.0 & 9.0 & 11.0 & 1.0 & 6.5 & 7.5 & 0.5 & 4.0 & 5.0 & 0 \\
\hline 3 & 8.0 & 11.5 & 1.0 & 7.09 & 9.5 & 1.0 & 11.0 & 15.0 & 0.25 & 5.0 & 7.0 & 0 & 7.0 & 7.0 & 1.0 & 5.0 & 6.0 & 1.0 & 5.0 & 6.5 & 0.33 \\
\hline 4 & 8.0 & 11.5 & 1.0 & 7.09 & 9.5 & 1.0 & 11.0 & 15.0 & 0.25 & 1.0 & 1.0 & 1.0 & 9.0 & 11.0 & 1.0 & 5.0 & 6.0 & 1.0 & 5.0 & 6.5 & 0.33 \\
\hline 5 & 8.0 & 11.5 & 0.86 & 7.09 & 9.5 & 1.0 & 11.0 & 15.0 & 0.63 & 5.0 & 7.0 & 0 & 7.0 & 7.0 & 1.0 & 6.5 & 7.5 & 0 & 3.0 & 3.5 & 0 \\
\hline 6 & 8.0 & 11.5 & 0.86 & 7.09 & 9.5 & 1.0 & 11.0 & 15.0 & 0.63 & 1.0 & 1.0 & 1.0 & 9.0 & 11.0 & 1.0 & 6.5 & 7.5 & 0 & 3.0 & 3.5 & 0 \\
\hline 7 & 8.0 & 11.5 & 0.71 & 7.09 & 9.5 & 0.6 & 8.0 & 13.5 & 0.18 & 4.75 & 6.75 & 0 & 6.75 & 6.75 & 1.0 & 6.75 & 7.75 & 0.5 & 3.75 & 5.25 & 0 \\
\hline 8 & 8.0 & 11.5 & 0.71 & 7.09 & 9.5 & 0.6 & 8.0 & 13.5 & 0.18 & 0.75 & 0.75 & 1.0 & 8.75 & 10.75 & 1.0 & 6.75 & 7.75 & 0.5 & 3.75 & 5.25 & 0 \\
\hline 9 & 8.0 & 11.5 & 0.71 & 7.0 & 9.5 & 0.6 & 8.0 & 13.5 & 0.18 & 4.75 & 6.75 & 0 & 6.75 & 6.75 & 1.0 & 5.25 & 6.25 & 1.0 & 4.75 & 6.75 & 0.25 \\
\hline 10 & 8.0 & 11.5 & 0.71 & 7.09 & 9.5 & 1.0 & 11.0 & 15.0 & 1.0 & 5.0 & 7.0 & 0 & 7.0 & 7.0 & 1.0 & 2.0 & 2.0 & 1.0 & 5.0 & 6.5 & 1.0 \\
\hline 11 & 8.0 & 11.5 & 0.71 & 7.0 & 9.5 & 0.6 & 8.0 & 13.5 & 0.18 & 0.75 & 0.75 & 1.0 & 8.75 & 10.75 & 1.0 & 5.25 & 6.25 & 1.0 & 4.75 & 6.75 & 0.25 \\
\hline 12 & 8.0 & 11.5 & 0.71 & 7.09 & 9.5 & 1.0 & 11.0 & 15.0 & 1.0 & 1.0 & 1.0 & 1.0 & 9.0 & 11.0 & 1.0 & 2.0 & 2.0 & 1.0 & 5.0 & 6.5 & 1.0 \\
\hline
\end{tabular}

Although the leader's satisfactory degree has dropped to 0.71 under the solution $\left(x^{12}, y_{1}^{12}, y_{2}^{12}, z_{11}^{12}, z_{12}^{12}, z_{21}^{12}, z_{22}^{12}\right)=(2,1,0.5,3,1,1,0.5)$, the leader cannot obtain a better objective value or a higher satisfactory degree by moving away from the vertex over the inducible region (IR) under the Stackelberg-Nash equilibrium among all decision entities. Also, it is noticeable that each follower and its counterparts have to achieve the Nash equilibrium when making their individual decisions, because their decisions are interactively affected by each other. Therefore, within the real-world case study, the Stackelberg-Nash solution $\left(x^{12}, y_{1}^{12}, y_{2}^{12}, z_{11}^{12}, z_{12}^{12}, z_{21}^{12}, z_{22}^{12}\right)=(2,1,0.5,3,1,1,0.5)$ is the optimal solution to the MFTL decision model (17), which means a final compromised result among all decision entities under the current decision context in the conglomerate enterprise. This MFTL hierarchical decision situation indicates that the leader may not achieve an individual optimal solution under the constraint region even though it has priority in making decisions, since its decisions are determined by implicit reactions of the followers. Moreover, the decision process and results of an MFTL decision problem are affected by the reference-uncooperative relationship among multiple followers at the same level. In summary, the proposed MFTL decision techniques provide an effective way to model and solve real-world MFTL decision problems and to recognize the satisfactory degree of decision entities towards solutions.

Furthermore, by the optimal solution, we can analyze whether or not the conglomerate employed practical and effective management strategies to balance the production-inventory planning among its subordinate sales company, logistics centers and manufacturing factories. Based on the given experimental data in Table 5 , the contrastive analysis between the upper limits to the holding inventory or overtime production capacity of each decision entity and the final solution is shown as Fig. 6. It can be seen from Fig. 6 that the holding 
inventories of the logistics centers peak at their respective upper limits. Also, the production outputs of the manufacturing factories 11 and 21 reach their maximum overtime production capacities respectively. In contrast, the holding inventory or overtime production outputs of other decision entities are less or much less than their corresponding upper limits. These results indicate that decision entities whose inventory holding cost or overtime production cost is lower prefer to keep more inventories or manufacture more production outputs under the current decision context, which is exactly desired by the conglomerate as presented in Section 5.1. Therefore, the current management strategy implemented by the conglomerate is an available way to balance the production-inventory planning throughout the three-stage supply chain with conflicting objectives of decision entities.

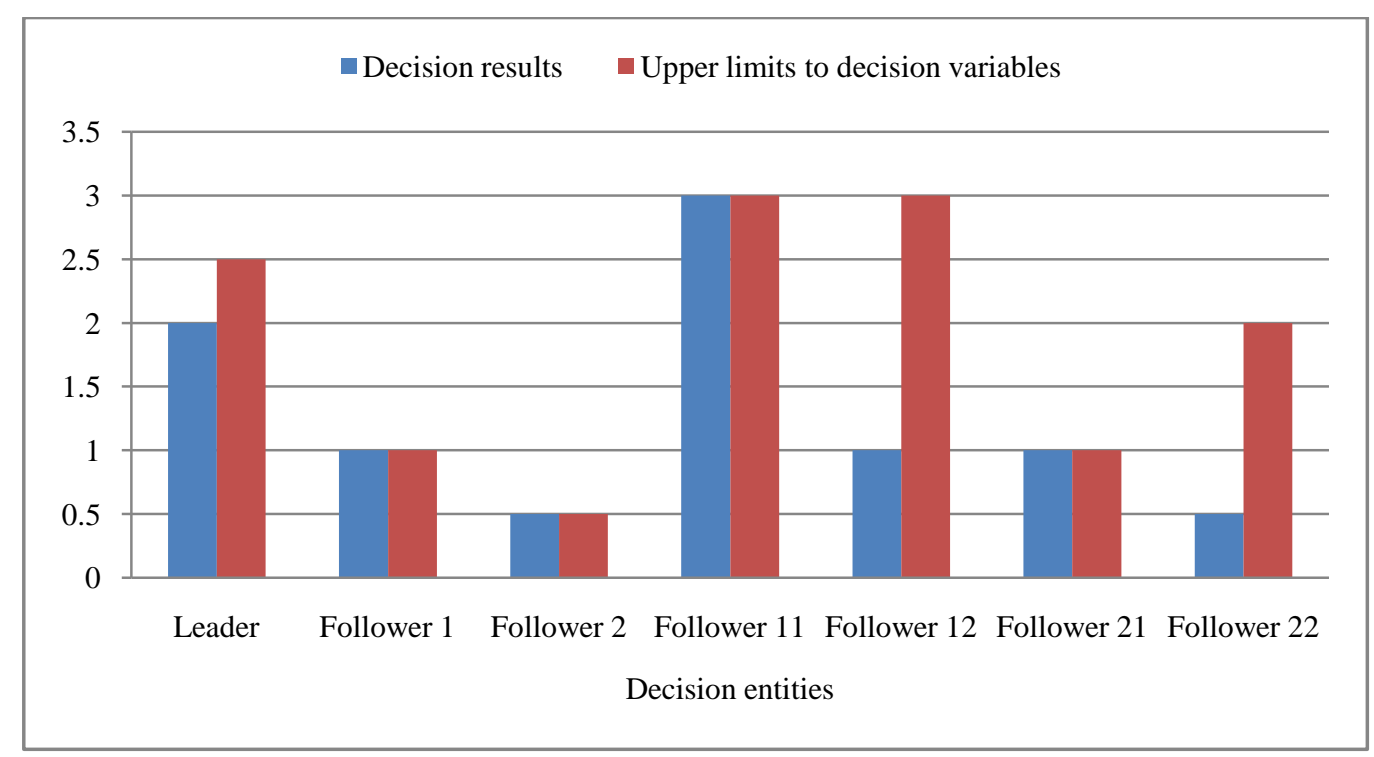

Fig. 6. The contrastive analysis of results

\subsection{Further discussions}

This section will discuss in depth characteristics of the MFTL Kth-Best algorithm and the evaluation criterion defined by fuzzy programming. Also, we will analyze limitations to our research and address future studies.

Table 8 clearly shows that we finally find an optimal solution by completing the enumeration of 12 vertices, of which most ( 8 in 12) are accompanied by the same decision made by the leader and the middle-level followers, which implies that the search approach of the MFTL Kth-Best algorithm is easily convergent. Also, only a few data involving $W_{k}, T$, and $W$ are necessary to write down within the algorithm operation. We can also observe the features of the algorithm through computing Example 1 in Section 3. Thus, the MFTL Kth-Best algorithm can be carried out efficiently because each successive pair of points is adjacent. Moreover, note that the other 11 vertices searched, apart from the optimal vertex 12, are all feasible solutions to the MFTL decision problem even if they cannot be an optimal solution. The property gives us another advantage of the MFTL Kth-Best algorithm in that the upper and lower bounds on an optimal solution are generated by the procedure even if storage or computational limits are reached before convergence. However, when plenty of followers are involved at the middle and bottom levels or a large 
number of decision variables and constraints exist, the execution efficiency of the algorithm may experience a steep decline as superabundant vertices are needed to complete the search. Our future research will explore the performance of the MFTL $K$ th-Best algorithm through sufficient numerical experiments.

It is noticeable from Tables 9 and 10 that the middle-level follower 2 obtains the same individual objective value 12.5 at the vertices $s^{5}$ and $s^{7}$; however, following this, the decision entity achieves two different satisfactory degrees 0.63 and 0.18 respectively. Also, note Table 3 in Section 4 that the objective value of the bottom-level follower 11 in Example 1 becomes worse from 7.5 to 9.0; however, following this, the corresponding satisfactory degree increases from 0.83 to 1.0. Evidently, it is not a positive correlation between the objective value and the corresponding satisfactory degree for followers. In this case study, the situation means that the feasible set and the rational set of the middle-level follower 2 are changed as the leader and the middle-level follower 1 change their decisions $\left(x, y_{1}\right)=(2,1)$ to $\left(x, y_{1}\right)=(2.5,0.5)$. Therefore, the satisfactory degree can be considered as a relative but not an absolute evaluation criterion as individual best and worst objective values of each decision entity would vary with the changing externalities determined by others, which clearly reflects the characteristic of the MFTL hierarchical decision-making process.

In this study, we focus on the reference-uncooperative relationship within a three-stage supply chain comprised of one leader and multiple followers. All decision entities have to achieve a Stackelberg-Nash solution under the current decision conditions within the three-stage supply chain. Thus, under the uncooperative situation, decision entities have to adjust the current decision context through changing objective functions or constraint conditions to generate a new round of decision-making processes if they desire to improve their respective satisfactory degrees. However, all decision entities that are distributed throughout a conglomerate enterprise may have chances to cooperate with each other and achieve an agreement on their decisions in the real world. For example, if the leader desires to improve its own satisfactory degree, it may persuade the middle-level follower 2 and the bottom-level follower 22 to react to others' decisions $\left(x, y_{1}, z_{11}, z_{12}, z_{21}\right)=(2,1,1,3,1)$ by determining their own decisions $\left(y_{2}, z_{22}\right)=(0.5,1.5)$ such that the leader can achieve its individual best solution, yielding the solution $\left(x^{4}, y_{1}^{4}, y_{2}^{4}, z_{11}^{4}, z_{12}^{4}, z_{21}^{4}, z_{22}^{4}\right)=(2,1,0.5,1,3,1,1.5)$. Thus, definitions of the satisfactory degree provide a practical way in finding some possibly satisfactory solutions but not just a Stackelberg-Nash solution to a MFTL decision case in the real world, because the satisfactory degree can be considered as an evaluation criterion that can be adopted to recognize a solution whether or not decision entities desire it. Also, the evaluation criterion provides an available approach to solve a MFTL decision problem without a Stackelberg-Nash solution. As we discussed above, if decision entities are willing to cooperate with each other, we can find a satisfactory solution through recognizing the satisfactory degree of decision entities. Our future research will extend the evaluation criterion defined by the fuzzy programming approach to handle real-world MFTL decision cases in which there does not exist a Stackelberg-Nash solution or decision entities prefer to cooperate with one another. 


\section{Conclusions and further study}

This paper presents our development to handle the reference-uncooperative MFTL decision problem: information exchange among multiple followers at the same level even though they are independent and uncooperative decision entities. Solving this decision problem needs to find an optimal solution achieving not only the Stackelberg equilibrium in the vertical structure but also the Nash equilibrium among multiple followers at the same horizontal level. The paper therefore first proposed a general MFTL decision model to describe the reference-uncooperative situation. It then developed a MFTL $K$ th-Best algorithm to find an optimal solution (also known as a Stackelberg-Nash solution) to the model based on related theoretical properties. Moreover, we evaluated the solution obtained and identified the satisfaction of decision entities using a fuzzy programming approach. Lastly, a real-world case study on production-inventory planning illustrated the effectiveness of the proposed MFTL decision techniques in handling such problems of applications. The results indicate that this paper provides a practical way to deal with reference-based uncooperative MFTL hierarchical decision-making problems from the perspective of theory and application. The limitation of this study is that the computational load of the MFTL Kth-Best algorithm may increase steeply with increase in the mass of variables and constraints. Thus, we will explore the execution efficiency of the algorithm through sufficient numerical experiments in our future study. We will also extend the evaluation criterion defined by the fuzzy programming approach to solve MFTL decision problems without Stackelberg-Nash solutions. In addition, we will focus our future research on other relationships, such as cooperative and semi-cooperative situations [18], among multiple followers in MFTL decision problems.

\section{Acknowledgements}

This work is supported by the Australian Research Council (ARC) under discovery grant DP140101366, the National High Technology Research and Development Program of China (NO. 2013AA040402).

\section{References}

[1] N. Alguacil, A. Delgadillo, J.M. Arroyo, A trilevel programming approach for electric grid defense planning, Computers \& Operations Research, 41 (2014) 282-290.

[2] R.G. Askin, I. Baffo, M. Xia, Multi-commodity warehouse location and distribution planning with inventory consideration, International Journal of Production Research, 52 (2014) 1897-1910.

[3] C. Audet, J. Haddad, G. Savard, A note on the definition of a linear bilevel programming solution, Applied Mathematics and Computation, 181 (2006) 351-355.

[4] J.F. Bard, An investigation of the linear three level programming problem, IEEE Transactions on Systems, Man, and Cybernetics, SMC-14 (1984) 711-717.

[5] J.F. Bard, Practical Bilevel Optimization: Algorithms and Applications, Kluwer Academic Publishers, Dordrecht, The Netherlands, 1998.

[6] P. Berling, J. Marklund, Multi-echelon inventory control: an adjusted normal demand model for implementation in practice, International Journal of Production Research, (2014) 1-17.

[7] H.K. Chan, F.T.S. Chan, Comparative study of adaptability and flexibility in distributed manufacturing supply chains, Decision Support Systems, 48 (2010) 331-341.

[8] Y.-C. Chang, V.C. Li, C.-J. Chiang, An ant colony optimization heuristic for an integrated production and distribution 
scheduling problem, Engineering Optimization, 46 (2014) 503-520.

[9] S.-W. Chiou, A bi-level programming for logistics network design with system-optimized flows, Information Sciences, 179 (2009) 2434-2441.

[10] S. Dempe, Foundations of Bilevel Programming, Kluwer Academic Publishers, Dordrecht, The Netherlands, 2002.

[11] N.P. Faísca, P.M. Saraiva, B. Rustem, E.N. Pistikopoulos, A multi-parametric programming approach for multilevel hierarchical and decentralised optimisation problems, Computational Management Science, 6 (2007) 377-397.

[12] S.R. Hejazi, A. Memariani, G. Jahanshahloo, M.M. Sepehri, Linear bilevel programming solution by genetic algorithm, Computers \& Operations Research, 29 (2002) 1913-1925.

[13] W.-T. Ho, Y.-C. Hsiao, An integrated production and inventory model for a system comprising an assembly supply chain and a distribution network, International Journal of Systems Science, 45 (2014) 841-857.

[14] H. Kucukaydin, N. Aras, I.K. Altinel, Competitive facility location problem with attractiveness adjustment of the follower: A bilevel programming model and its solution, European Journal of Operational Research, 208 (2011) 206-220.

[15] Y.-J. Lai, Hierarchical optimization: A satisfactory solution, Fuzzy Sets and Systems, 77 (1996) 321-335.

[16] D. Li, J.B. Cruz Jr, Information, decision-making and deception in games, Decision Support Systems, 47 (2009) 518-527.

[17] J. Lu, C. Shi, G. Zhang, On bilevel multi-follower decision making: General framework and solutions, Information Sciences, 176 (2006) 1607-1627.

[18] J. Lu, G. Zhang, J. Montero, L. Garmendia, Multifollower trilevel decision making models and system, IEEE Transactions on Industrial Informatics, 8 (2012) 974-985.

[19] A.G. Mersha, S. Dempe, Linear bilevel programming with upper level constraints depending on the lower level solution, Applied Mathematics and Computation, 180 (2006) 247-254.

[20] S. Mitiku, A multilevel programming approach to decentralized (or hierarchical) resource allocation systems, Proceedings in Applied Mathematics and Mechanics, 7 (2007) 2060003-2060004.

[21] S. Pramanik, T.K. Roy, Fuzzy goal programming approach to multilevel programming problems, European Journal of Operational Research, 176 (2007) 1151-1166.

[22] S.S. Sana, A production-inventory model of imperfect quality products in a three-layer supply chain, Decision Support Systems, 50 (2011) 539-547.

[23] H.-S. Shih, Y.-J. Lai, E.S. Lee, Fuzzy approach for multi-level programming problems, Computers \& Operations Research, 23 (1996) 73-91.

[24] S. Sinha, Fuzzy mathematical programming applied to multi-level programming problems, Computers \& Operations Research, 30 (2003) 1259-1268.

[25] S. Sinha, Fuzzy programming approach to multi-level programming problems, Fuzzy Sets and Systems, 136 (2003) 189-202.

[26] H.V. Stackelberg, The Theory of Market Economy, Oxford University Press, Oxford, 1952.

[27] A. Street, A. Moreira, J.M. Arroyo, Energy and reserve scheduling under a joint generation and transmission security criterion: An adjustable robust optimization approach, IEEE Transactions on Power Systems, 29 (2014) 3-14.

[28] P.S. Tan, S.S.G. Lee, A.E.S. Goh, Multi-criteria decision techniques for context-aware B2B collaboration in supply chains, Decision Support Systems, 52 (2012) 779-789.

[29] S.A. Torabi, M. Ebadian, R. Tanha, Fuzzy hierarchical production planning (with a case study), Fuzzy Sets and Systems, 161 (2010) 1511-1529.

[30] L. Vicente, P. Calamai, Bilevel and multilevel programming: A bibliography review, Journal of Global Optimization, 5 (1994) 291-306.

[31] Z. Wan, L. Mao, G. Wang, Estimation of distribution algorithm for a class of nonlinear bilevel programming problems, Information Sciences, 256 (2014) 184-196.

[32] D.J. White, Penalty function approach to linear trilevel programming, Journal of Optimization Theory and 
Applications, 93 (1997) 183-197.

[33] X. Xu, Z. Meng, R. Shen, A tri-level programming model based on Conditional Value-at-Risk for three-stage supply chain management, Computers \& Industrial Engineering, 66 (2013) 470-475.

[34] Y. Yao, T. Edmunds, D. Papageorgiou, R. Alvarez, Trilevel optimization in power network defense, IEEE Transactions on Systems, Man, and Cybernetics, 37 (2007) 712-718.

[35] D. Zhang, G.-H. Lin, Bilevel direct search method for leader-follower problems and application in health insurance, Computers \& Operations Research, 41 (2014) 359-373.

[36] G. Zhang, J. Lu, J. Montero, Y. Zeng, Model, solution concept, and Kth-best algorithm for linear trilevel programming, Information Sciences, 180 (2010) 481-492. 